\title{
High Robustness and Reliability of Fuzzy Logic Based Position Estimation for Sensorless Switched Reluctance Motor Drives
}

\author{
Adrian David Cheok, Member, IEEE, and Nesimi Ertugrul, Member, IEEE
}

\begin{abstract}
In many applications where motor drives are used, reliability is of high concern. Thus, a major consideration is the reliability of position estimation schemes when sensorless SR motor drive control is employed. Hence, in this paper, the robust operation of a fuzzy logic based angle estimation algorithm for the switched reluctance motor (SR) motor is described. It is shown using theoretical analysis and experimental results, that by using fuzzy logic, the angle estimation scheme gains a high level of robustness and reliability. A theoretical and quantitative analysis of the noise and error commonly found in practical motor drives is given, and how this can affect SR motor position estimation. An analysis is also given on the concepts of robustness and reliability. It is shown that the fuzzy logic based scheme is robust to erroneous and noisy signals commonly found in motor drives.
\end{abstract}

Index Terms-Error analysis, estimation, fuzzy logic, fuzzy systems, modeling, nonlinear estimation, reluctance motor drives.

\section{INTRODUCTION}

$\mathbf{T}$ HE SWITCHED reluctance (SR) motor has advantages in practical variable speed drive systems due to an intrinsic simplicity and ruggedness that makes it well suited for many commercial applications. Generally the SR drive controller requires rotor position feedback, because excitation of the SR motor phases need to be synchronized with the rotor angle. Position sensors are commonly employed to obtain rotor position measurements, however in many systems advantages can be found in eliminating these sensors. These benefits include the elimination of electrical connections to the sensors, reduced size, low maintenance, and insusceptibility to environmental factors.

Hence, a diverse range of indirect or sensorless position estimation methods has previously been proposed. These have been extensively detailed and reviewed previously [1]-[3]. The previous estimation methods can be classified into two major groups. In one group, low amplitude test signals are inserted into the motor phase windings to derive rotor position information, whilst in the other group the actual motor excitation waveforms are monitored without the use of additional signals in the motor. Mathematical model based schemes are also classified under the latter group.

Manuscript received July 23, 1998; revised July 23, 1999. Recommended by Associate Editor, M. Arefeen.

A. D. Cheok is with the Department of Electrical Engineering, National University of Singapore, Singapore 119260 (e-mail: adriancheok@ nus.edu.sg).

N. Ertugrul is with the Department of Electrical and Electronic Engineering, University of Adelaide, Adelaide 5005, Australia (e-mail: nesimi@eleceng.adelaide.edu.au).

Publisher Item Identifier S 0885-8993(00)02326-7.
A major concern of position estimation using these schemes is that feedback signals measured from the motor are required to calculate the motor position. Therefore, the accuracy of the estimated position is dependent on the accuracy of the measured feedback signals. However, motor drives are electromagnetically noisy environments, and in addition practical measurement circuits of electronic signals are imperfect. Thus, the feedback signals are generally corrupted by noise and errors in practical motor drives.

Hence, a rarely stated disadvantage of sensorless position estimation as opposed to sensor based position estimation (using such devices as incremental or absolute encoders), is that the sensors have a guaranteed accuracy under normal conditions, and usually a high reliability. Furthermore, the sensors are very robust to electromagnetic noise (for example, the feedback signals from position encoders are usually TTL logic level signals).

Therefore, although it is commonly stated that position sensors tend to reduce the reliability of a drive system, the previously developed estimation schemes may not be useful in industrial applications unless their reliability and robustness against noise and error is proven.

Thus in this paper, the high robustness and reliability of a new rotor position estimator for the SR motor is described. The position estimator uses fuzzy logic based modeling, estimation, and prediction in a novel manner to provide a high system reliability against feedback signal noise and error.

The first part of this paper will analyze the noise and error commonly found in practical motor drives, and it will be shown that the feedback noise affects the accuracy of rotor position estimation. Secondly, the new fuzzy logic based position estimation algorithm will be described, and a theoretical analysis of the robustness of the scheme will be given. Finally experimentally based results will be shown with the fuzzy logic based position estimator operating with erroneous and noisy signals due to factors including electromagnetic noise, low sampling frequencies, and flux linkage offset error.

\section{NOISE AND ERROR IN SR MOTOR POSITION ESTIMATION}

As discussed in [2], essentially all position estimation schemes for the SR motor are based on the relationship of the motor phase magnetic flux linkage with rotor position. The measured flux linkage characteristics of an SR motor (described in Appendix A) are shown in Fig. 1. In the figure, zero degrees represents the aligned rotor position and thirty 


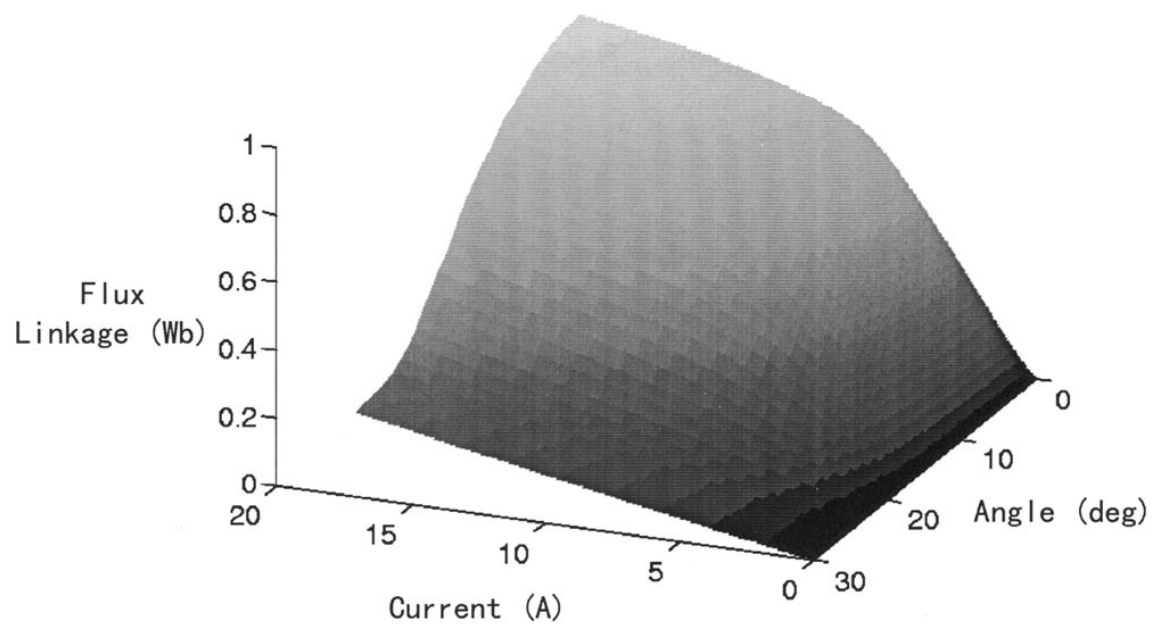

Fig. 1. Measured magnetization characteristics of SR motor (as flux linkage varies from 0 to 1 , surface color changes from dark to light).

TABLE I

SOURCES OF FEEDBACK ERROR

\begin{tabular}{l|l}
\hline Measurement Errors & $\begin{array}{l}\text { a) Sensor offset error. } \\
\text { b) Sensor scaling error. }\end{array}$ \\
\hline Parameter Variation & $\begin{array}{l}\text { a) Variation of motor phase winding resistance value, primarily due to temperature } \\
\text { variation. }\end{array}$ \\
\hline Measurement Noise & $\begin{array}{l}\text { a) Noise due to capacitive coupling of measuring circuits and associated cabling with } \\
\text { switching power semiconductor circuit. } \\
\text { b) Electromagnetic interference from motor and power circuit on the measuring cir- } \\
\text { cuit. } \\
\text { c) Noise in measuring circuits (including (A/D) converter circuits) due to non-ideal } \\
\text { circuit layout and grounding. } \\
\text { d) Noise in A/D circuits due to noise generated from any connected microprocessor } \\
\text { digital bus lines. } \\
\text { e) Crosstalk between signals due to coupling in signal cabling. }\end{array}$ \\
\hline Integration Errors & $\begin{array}{l}\text { a) Drift and temperature variation (analog integrators). } \\
\text { b) Integration offet errors (analog and digital integration). } \\
\text { c) Time quantization errors due to finite sampling period (digital integration). } \\
\text { d) Amplitude quantization errors due to A/D conversion. }\end{array}$ \\
\hline
\end{tabular}

degrees represents the unaligned position. It can clearly be seen that the flux linkage $\psi(\theta, i)$ is a non-linear function of rotor position $\theta$ and current $i$. Therefore, if the flux linkage can be determined, together with current measurement, then the rotor position may be estimated. Furthermore, inductance $L(\theta)$ (which can be derived from the flux linkage if it is assumed that the motor magnetic circuit is linear) can also be used to determine position.

Existing literature on the effects of errors in previously developed SR motor position estimation schemes has been relatively sparse. In [4] only the time and amplitude quantization errors due to digital implementation of a specific linear inductance based scheme was discussed. The issue was further discussed in [5], where specific types of SR motor physical designs were proposed to improve the robustness of position estimation to error. However for simplicity all error analysis were based on linear or piecewise-linear motor magnetization characteristics.
In this error analysis no assumptions will be made about the motor linearity, and thus the results may be applied to any sensorless position estimation scheme in general.

Hence, consider the estimation of position using the nonlinear magnetization function $\psi(\theta, i)$, from measured values of flux linkage $\psi$ and current $i$. Generally flux linkage is impractical to measure directly, and thus integration of the total voltage across an excited phase is a common method to estimate phase flux

$$
\psi=\int(v-i R) d t
$$

where

$\psi$ phase flux linkage;

$R \quad$ phase resistance;

$v$ phase voltage;

$i$ phase current. 
TABLE II

ANALYTICAL ERROR EXPRESSIONS

\begin{tabular}{|c|c|}
\hline $\begin{array}{l}\text { 1. Due to dc offset in voltage } \\
\text { and current measurements }\end{array}$ & $\Delta \psi_{o}(k)=k \Delta T\left(v_{\circ}-R i_{o}\right)$ \\
\hline $\begin{array}{l}\text { 2. Due to amplitude or scaling } \\
\text { error }\end{array}$ & $\Delta \psi_{e}(k)=\sum_{n=0}^{k-1} \Delta T\left(A_{v}(v(n)+v(n-1))-R A_{i}(i(n)+i(n-1))\right) / 2$ \\
\hline $\begin{array}{l}\text { 3. Due to time quantization of } \\
\text { A/D converters }\end{array}$ & $\Delta \psi_{t q}(k)=\frac{k \Delta T P^{2} M}{12}$ \\
\hline $\begin{array}{l}\text { 4. Due to amplitude quantiza- } \\
\text { tion of } A / D \text { converters }\end{array}$ & $-k \Delta T\left(R \frac{2 I_{\max }}{2^{n}-1}\right) \leq \Delta \psi_{a q}(k) \leq k \Delta T\left(\frac{2 V_{\max }}{2^{n}-1}\right)$ \\
\hline 5. Due to noise & $\Delta \psi_{\mu}(k)=k \Delta T\left(\mu_{v}-R \mu_{i}\right)$ \\
\hline 6. Due to resistance variation & $\Delta \psi_{r}(k)=k \Delta T \Delta R \sum_{n=0}^{k-1}(i(n)+i(n-1)) / 2$ \\
\hline
\end{tabular}

where: $v_{0}=$ voltage measurement dc offset error, $i_{0}=$ current measurement dc offset error, $A_{v}=$ voltage measurement amplitude error, $A_{i}=$ current measurement amplitude error, $P=\mathrm{A} / \mathrm{D}$ sample period, $M=$ highest value of second derivative of measured signals, $I_{\max }=$ maximum amplitude of $\mathrm{A} / \mathrm{D}$ converter current measurement, $V_{\max }=$ maximum amplitude of $\mathrm{A} / \mathrm{D}$ converter voltage measurement, $n=$ number or $\mathrm{A} / \mathrm{D}$ converter bits, $\mu_{v}=$ mean level of voltage feedback noise, $\mu_{i}=$ mean level of current feedback noise.

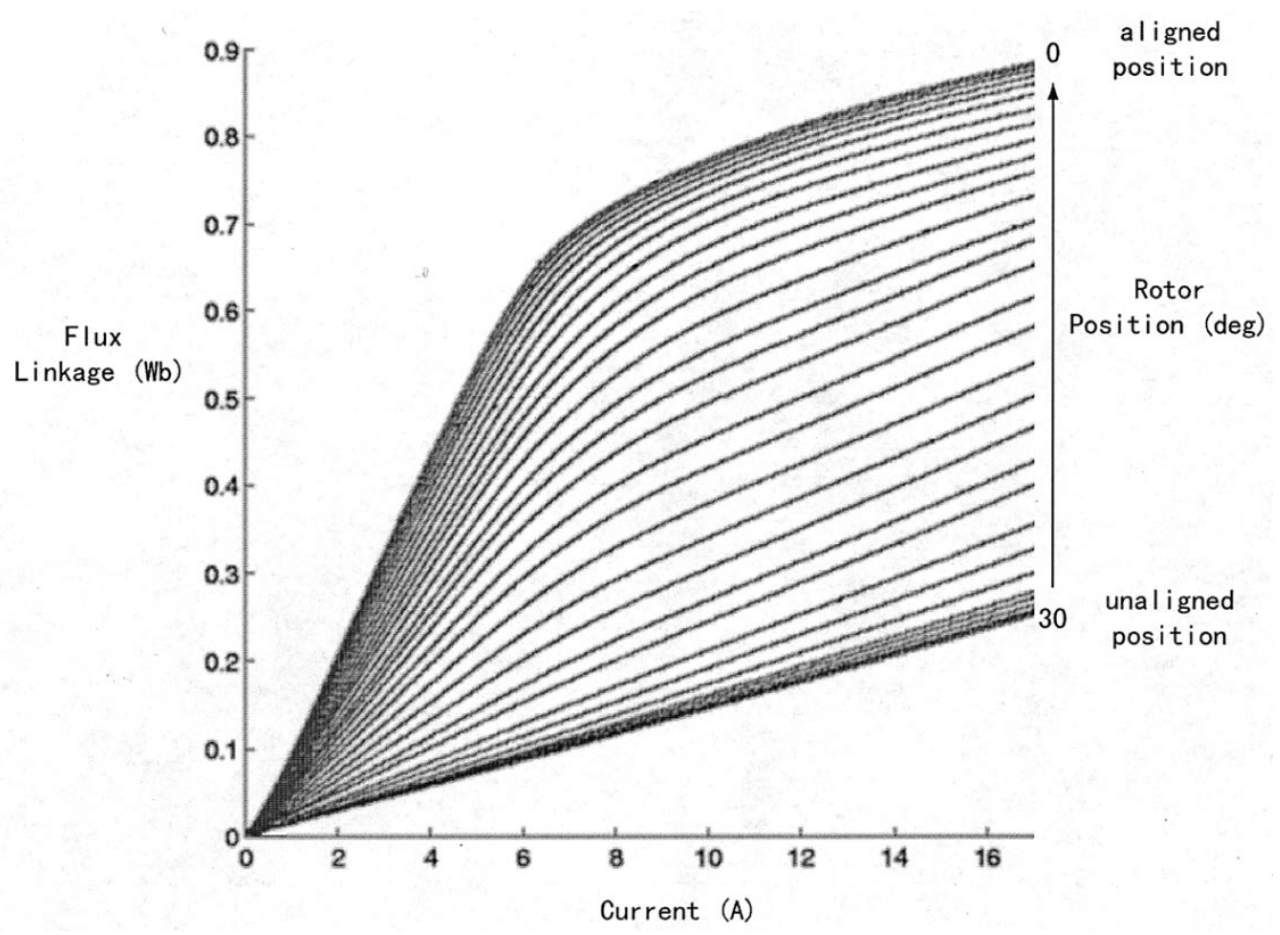

Fig. 2. Flux linkage versus current curves for SR motor.

Analog integrators can be used to estimate the flux linkages of the motor but these often have the problem of drift in the output signal due to temperature sensitivity and the need for compensation [6]. Therefore the integration may be performed digitally as shown in (2). This requires digitized phase voltage and current signals that are measured using analog to digital (A/D) converters

$$
\begin{aligned}
& \psi(n+1)= \\
& \psi(n)+\Delta T(v(n)-R i(n)+v(n-1)-R i(n-1)) / 2 \\
& \psi(0)=0
\end{aligned}
$$

where $n=$ sample number and $\Delta T=$ sampling period.
Hence, the feedback signals that are used for the estimation of rotor position in practice are the measured motor currents and voltage, and estimated or calculated flux linkage.

Generally, the feedback signals will contain some error component which can be defined as the difference between the actual physical motor quantities of current, voltage, and flux linkage, and the corresponding feedback values used for position estimation. The error components can be expressed as

$$
\begin{aligned}
\Delta v & =v_{m}-v \\
\Delta i & =i_{m}-i \\
\Delta \psi & =\psi_{e}-\psi
\end{aligned}
$$

where $\Delta v, \Delta i$, and $\Delta \psi$ are the error components, $v_{m}, i_{m}, a n d \psi_{e}$ are the measured and estimated values, $v$ is 


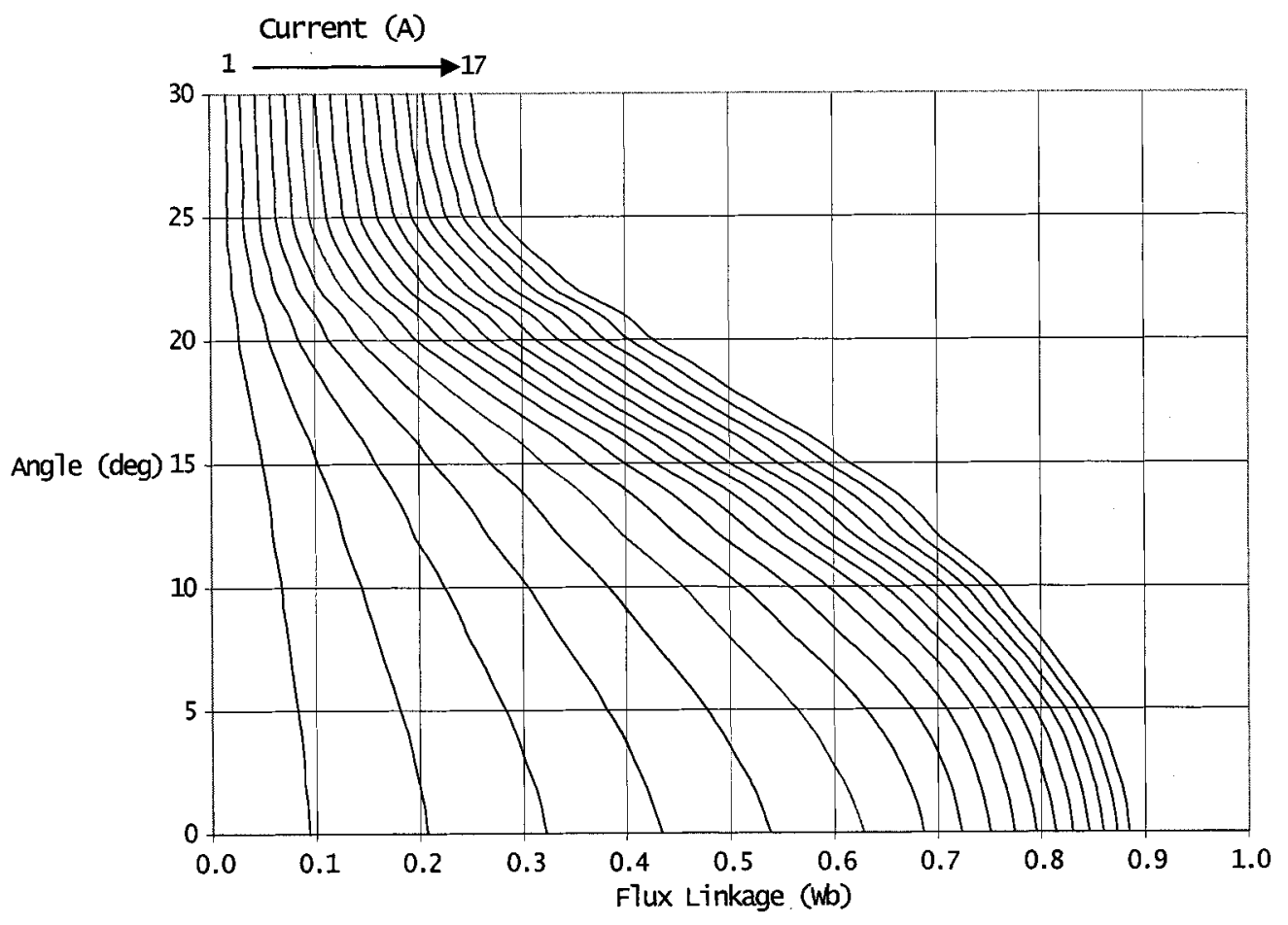

(a)

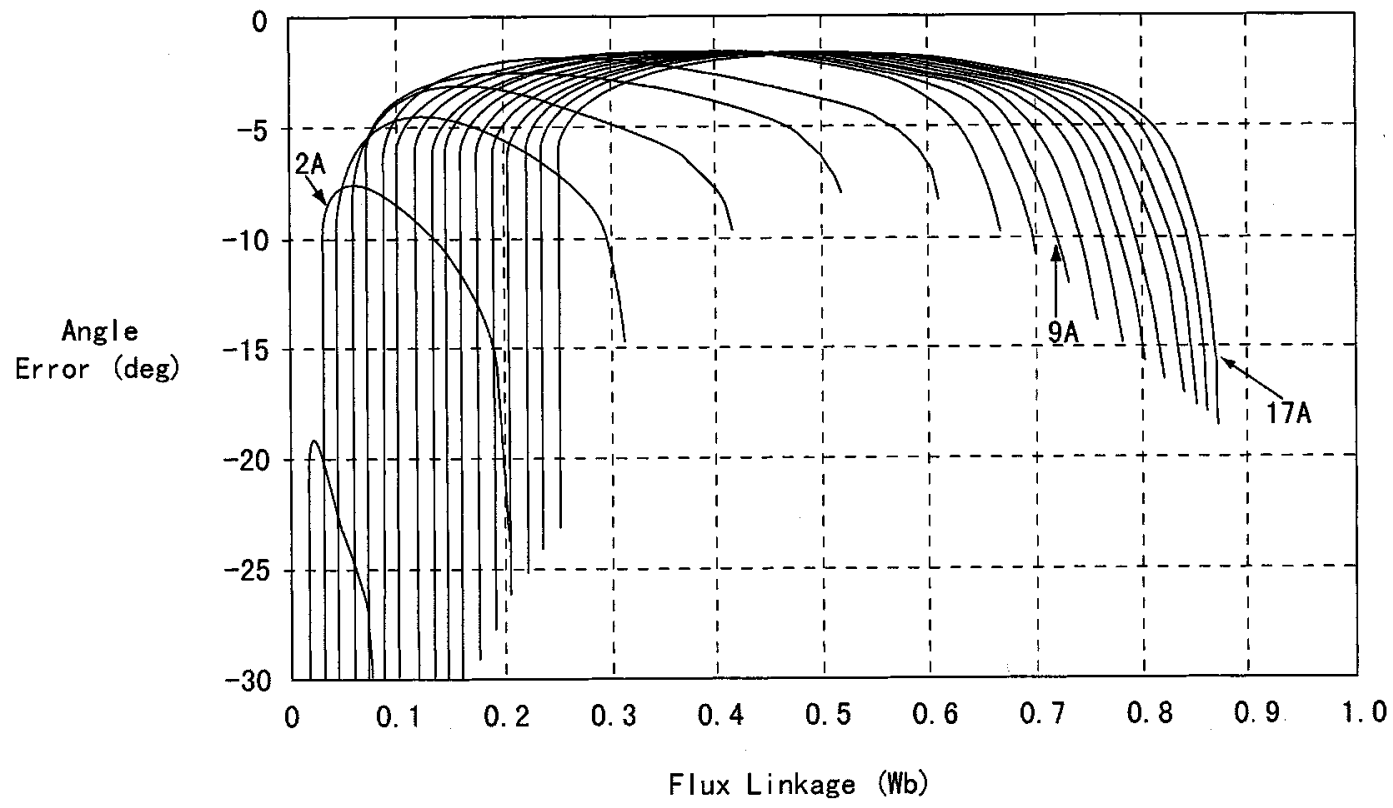

(b)

Fig. 3. (a) Rotor angle versus flux linkage and (b) angle error for $10 \%$ flux linkage feedback error.

with physical value of voltage in the motor, $i$ is the physical value of current in the motor, and $\psi$ is the physical value of flux linkage in the motor.

The source of the feedback error components in practical motor drives can be classified under various groups, as shown in Table I.

Using these classifications, an expression for the total flux linkage error due to the various error producing sources can be found. The total flux linkage error at some sample $k$ will be equal to

$$
\begin{aligned}
\Delta \psi(k)= & \Delta \psi_{o}(k)+\Delta \psi_{a}(k)+\Delta \psi_{t q}(k) \\
& +\Delta \psi_{a q}(k)+\Delta \psi_{\mu}(k)+\Delta \psi_{r}(k)
\end{aligned}
$$

where

$\Delta \psi$ total flux linkage error;

$\Delta \psi_{0}$ offset error;

$\Delta \psi_{a}$ amplitude error; 
TABLE III

Minimum ANGLE ESTIMATION ERROR AMPLITUDE FOR + $10 \%$ ERROR IN FLUX LINKAGE FEEDBACK VALUES

\begin{tabular}{c|c|c}
\hline Current $(\mathrm{A})$ & Error (deg) & Error (\%) \\
\hline 1 & 19.16 & 63.87 \\
2 & 7.40 & 24.65 \\
3 & 4.59 & 15.29 \\
4 & 3.34 & 11.14 \\
5 & 2.57 & 8.57 \\
6 & 2.17 & 7.24 \\
7 & 1.99 & 6.56 \\
8 & 1.84 & 6.13 \\
9 & 1.78 & 5.93 \\
10 & 1.74 & 5.81 \\
11 & 1.73 & 5.79 \\
12 & 1.73 & 5.79 \\
13 & 1.72 & 5.74 \\
14 & 1.72 & 5.74 \\
15 & 1.71 & 5.72 \\
16 & 1.70 & 5.67 \\
17 & 1.74 & 5.81 \\
\hline
\end{tabular}

$\Delta \psi_{t q}$ time quantization error;

$\Delta \psi_{a q}$ amplitude quantization error;

$\Delta \psi_{\mu}$ mean signal noise error;

$\Delta \psi_{r}$ error due to change in resistance.

In [5] and [7], the analytical expressions of the various flux linkage error components in (7) were given. For reference, Table II summarizes some of the results.

If there are flux linkage estimation errors due to one or more of the above explained effects, there will be a corresponding error in the rotor position estimation. Regardless of the various sources of the total flux linkage estimation error expressed in (7), the corresponding angle error at sample time $k$ will be given by

$$
\Delta \theta(k)_{\Psi}=\left.\Delta \psi(k) \frac{\partial \theta}{\partial \psi}\right|_{i(k), \psi(k)}
$$

where

$\Delta \theta(k)_{\Psi}$ the angle error due to error in the flux linkage feedback;

$i(k) \quad$ the actual motor phase current;

$\psi(k) \quad$ the actual motor phase flux linkage.

Therefore, for a given flux error, the angle error will be proportional to the partial derivative of the rotor position function with respect to flux linkage at the point $(i(k), \psi(k))$.

Although quantitative analysis of this error will be detailed below, it is interesting to examine the angle error qualitatively. In Fig. 2 the magnetization characteristics of Fig. 1 are plotted on a two-dimensional plane with curves of constant rotor position. From the curves, it can be seen that when the angle is near the aligned position, the unaligned position, or the motor phase current is low, the angle curves are tightly bunched up. This entails that the slope of the function in the three-dimensional plane is high. Hence in these areas, the partial derivative of angle with respect to flux will be high. Under these conditions, according to (8), small errors in the flux linkage will result in large errors in the position estimate.
As was discussed above, the current feedback signal is also affected by measurement error and noise. Similarly to (8) the current feedback error at sample $k$ can be written as

$$
\Delta i(k)=\Delta i_{o}(k)+\Delta i_{a}(k)+\Delta i_{\mu}(k)
$$

where

$\Delta i(k) \quad$ total current feedback error;

$\Delta i_{o}(k)$ current measurement offset error;

$\Delta i_{a}(k) \quad$ current measurement amplitude error;

$\Delta i_{\mu}(k)$ current measurement mean signal noise.

Hence, even if the flux linkage could somehow be measured without error, the current measurement error will lead to a corresponding rotor position estimation error. For a given current error at sample time $k$ the angle error can be written as

$$
\Delta \theta(k)_{I}=\left.\Delta i(k) \frac{\partial \theta}{\partial i}\right|_{i(k), \psi(k)}
$$

where $\Delta \theta(k)_{I}=$ the angle error due to error in the current feedback.

By examining again the contour curves of angle with respect to current and flux (Fig. 2) it can be seen that similarly to the case of flux linkage error, the angle estimation error is most sensitive to current feedback error near the aligned and unaligned rotor positions and when the motor current is low. This results in the fact that in these areas the partial derivative of angle with respect to current will be high.

Generally there will be errors in both the flux linkage and current feedback. To consider the effect of simultaneous flux linkage and current errors on the position estimation the following expression may be employed

$$
\Delta \theta(k)=\left.\Delta \psi(k) \frac{\partial \theta}{\partial \psi}\right|_{i(k), \psi(k)}+\left.\Delta i(k) \frac{\partial \theta}{\partial i}\right|_{i(k), \psi(k)} .
$$

\section{A. Quantitative Error Analysis}

It was seen in the above analysis [(7), (9), and Table II] that the instantaneous feedback error of the flux linkage and current signals are complex and nonlinear functions. Furthermore, it was seen that to numerically calculate the exact instantaneous error requires detailed knowledge of the hardware parameters (e.g., offset error, amplitude error, resistance variation) which may be time and temperature variant. Additionally, the signal parameters will also need to be known (e.g., mean noise, measured signal time-derivatives) which are generally time varying and system dependent.

Therefore, to perform a quantitative error analysis it is normally not useful or feasible to predict the instantaneous feedback errors $\Delta \psi(k)$ and $\Delta i(k)$. Instead it will be useful to quantitatively analyze the resultant position error $\Delta \theta(k)$ for given feedback signal error amplitudes, regardless of their source, using (8) and (10).

1) Effect of Flux Linkage Error on Rotor Position Error: To examine the effect of flux linkage error on the rotor position estimation, the rotor position as a function of flux linkage at various values of constant current is plotted, as shown in Fig. 3(a). 


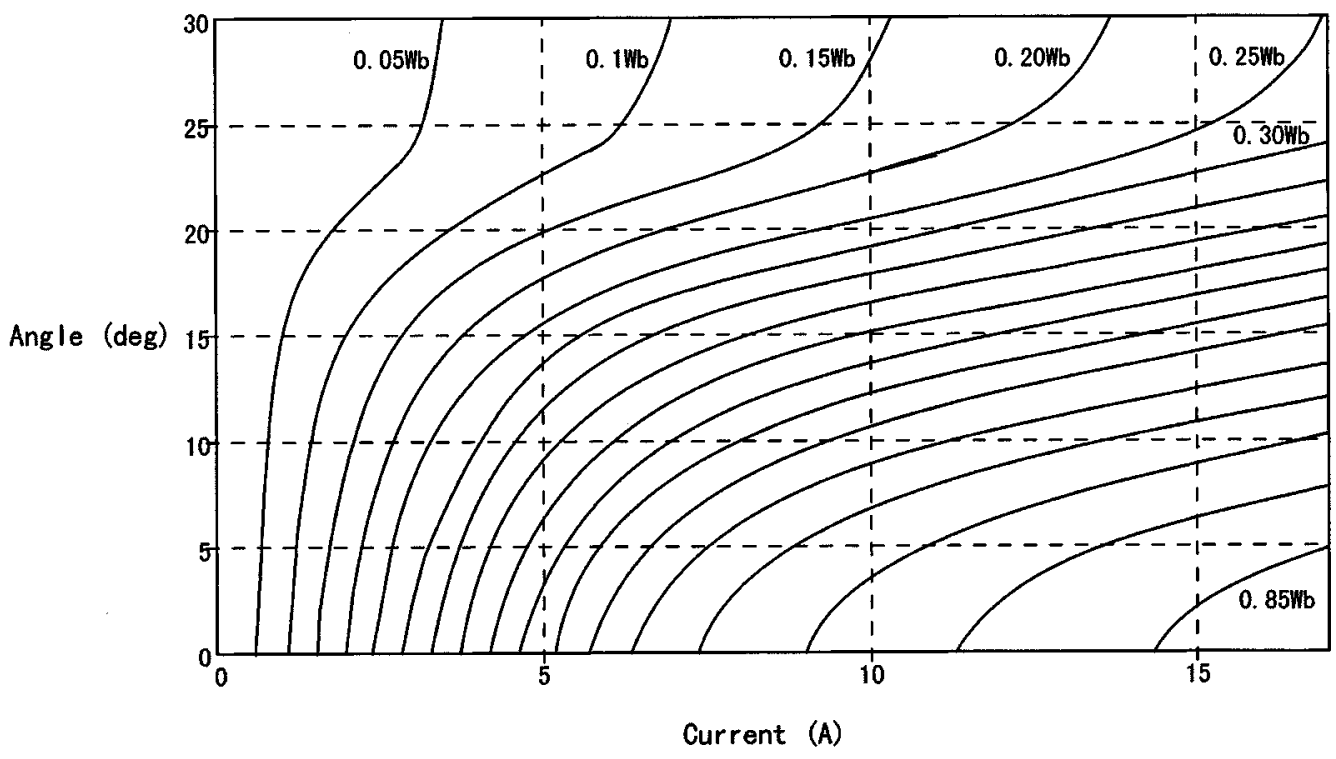

(a)

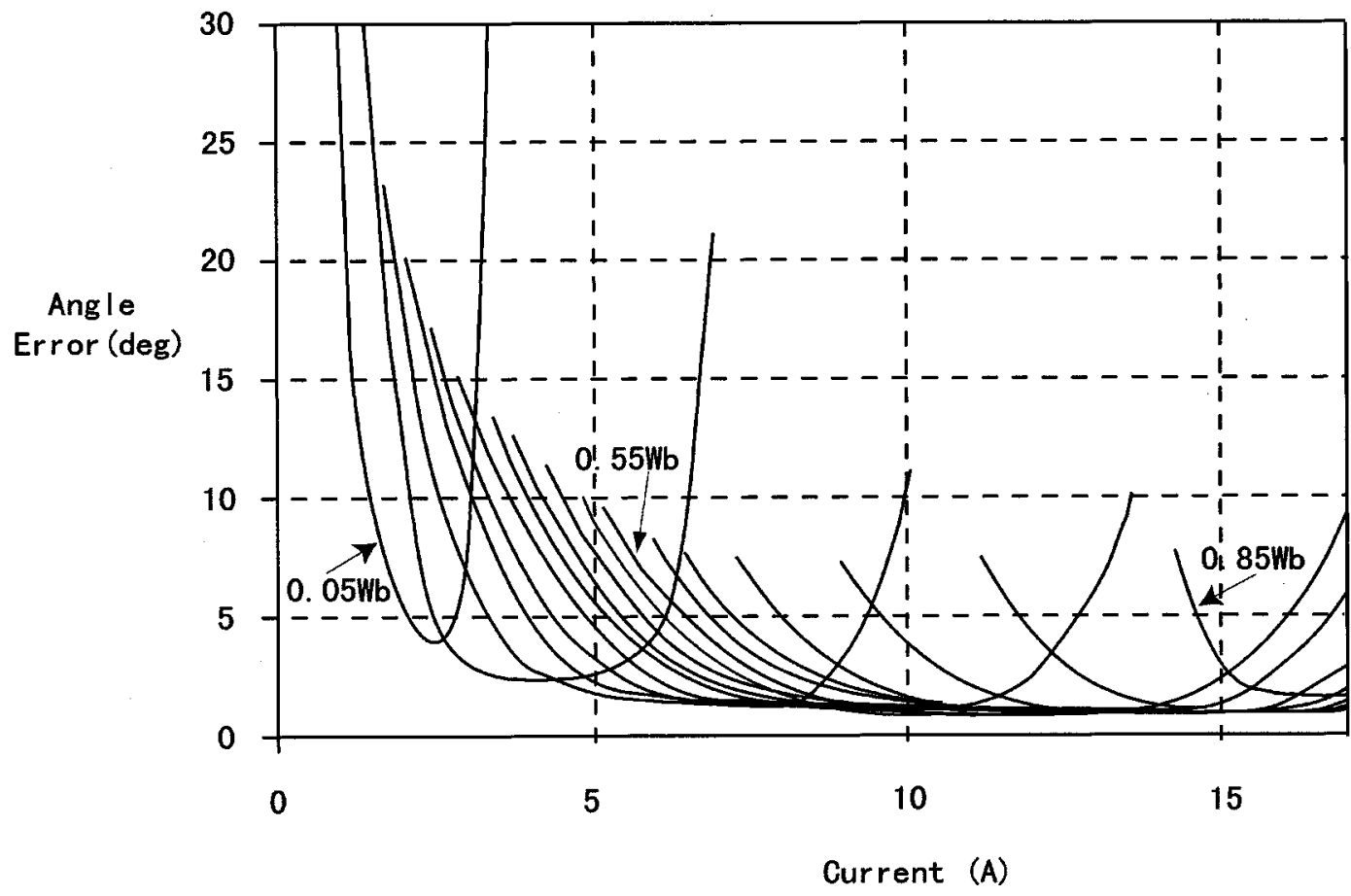

(b)

Fig. 4. (a) Rotor angle versus current, (b) angle error for $10 \%$ current feedback error.

These curves are derived from the motor magnetization characteristics previously shown in Fig. 1.

From the functions shown in Fig. 3(a), the function $\left.(\partial \theta / \partial \psi)\right|_{(i, \psi)}$ can be found. Hence, from the partial derivative functions, and using (8), the quantitative value of position error for a given error in the flux linkage estimate can be calculated.

As an example consider a flux linkage error that has an amplitude of $+10 \%$ of the maximum operating flux linkage $(0.7 \mathrm{~Wb})$ of the SR motor used in the research. Fig. 3(b) plots the calculated numerical value of the resultant angle estimation error.
It can be confirmed from the curves that, as discussed above, at low current, or near the aligned and unaligned angles, the angle estimation is more sensitive to errors in the flux linkage feedback. Fundamentally, this is due to the fact that the partial derivative, or slope of the curves shown in Fig. 3(a), is higher in these regions. It can also be noted that as the function $\left.(\partial \theta / \partial \psi)\right|_{(i, \psi)}$ is negative, the resultant angle error for positive flux error is negative, and vice-versa. When the rotor angle error is negative, this entails that the estimated angle lags the actual rotor angle of the motor. 
TABLE IV

MiNIMUM ANGLE ESTIMATION ERROR AMPLITUDE FOR $+10 \%$ ERROR IN CURRENT FEEDBACK VALUES

\begin{tabular}{c|c|c}
\hline Flux (Wb) & Error (deg) & Error (\%) \\
\hline 0.05 & 4.70 & 15.6 \\
0.10 & 2.50 & 8.3 \\
0.15 & 1.62 & 5.43 \\
0.20 & 1.25 & 4.17 \\
0.25 & 1.08 & 3.61 \\
0.30 & 0.96 & 3.21 \\
0.35 & 0.79 & 2.66 \\
0.40 & 0.80 & 2.68 \\
0.45 & 0.89 & 2.98 \\
0.50 & 0.91 & 3.03 \\
0.55 & 0.93 & 3.10 \\
0.60 & 0.97 & 3.23 \\
0.65 & 1.12 & 3.73 \\
0.70 & 1.14 & 3.80 \\
0.75 & 1.22 & 4.08 \\
0.80 & 1.32 & 4.41 \\
0.85 & 2.17 & 7.24 \\
\hline
\end{tabular}
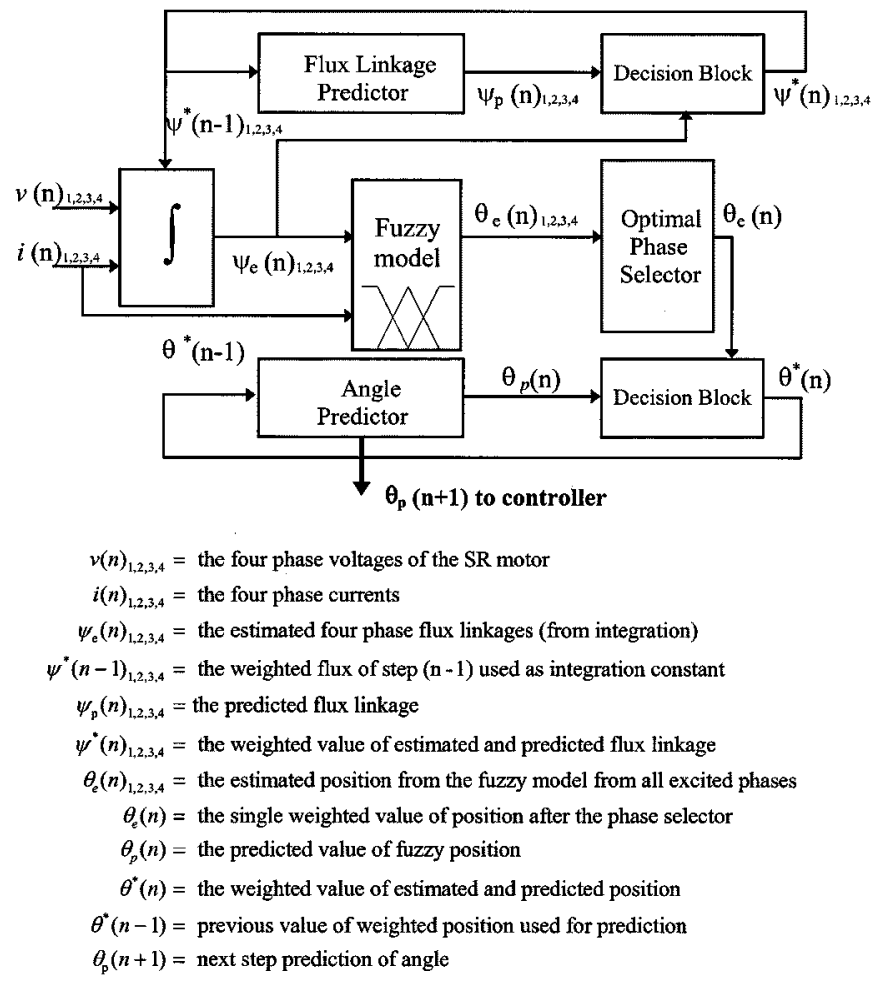

Fig. 5. The complete block diagram of the FL based position estimation algorithm.

The minimum resultant error amplitude for a $+10 \%$ error in the flux linkage for each of the curves shown in Fig. 3(a) is shown in Table III. The results are shown in both absolute degrees, and as a percentage of an electrical cycle of $30^{\circ}$. It should be emphasized that, as seen in Fig. 3(b), these are the minimum error values corresponding to the points when the derivative or slopes of the curves in Fig. 3(a) are at their minimum. However, at other points in the magnetization region, the derivative of the functions, and the corresponding angle error can be much higher.

2) Effect of Current Measurement Error on Rotor Position Error: To examine the effect of current feedback error on the rotor position estimation, the rotor position is defined as a function of current for constant values of flux linkage. The resultant curves, which are derived from the motor magnetization characteristics (Fig. 1), are shown in Fig. 4(a).

From the resultant curves, the partial derivative function $\left.(\partial \theta / \partial i)\right|_{(i, \psi)}$ can be calculated. Therefore using $(10)$, the quantitative value of position error for a given value of current error may be found. Similarly to the previous example above, position error values are calculated for a current amplitude error of $+10 \%$ of the maximum operating motor current (17 A). The corresponding position error curves are plotted in Fig. 4(b). From the curves it can be confirmed that, as discussed above, there is more sensitivity to error when the current is low and the angle is close to the aligned or unaligned positions.

The minimum position error for each of the curves is detailed in Table IV. Once again it should be noted that these points correspond to the points when the value of derivative of the curves shown in Fig. 4(a) are minimum. However, as can be seen from the resultant functions, the derivative and corresponding angle error at other points can be significantly higher.

It should be noted from the above results that for the same percentage error in the flux linkage and current feedback values, the resultant rotor position estimation error is generally lower for the case of current feedback error.

\section{Fuzzy Logic Based Position Estimation Scheme}

From the above discussion it was seen that the accuracy and reliability of sensorless position estimation is directly affected by feedback signal measurement noise and error. Therefore a new fuzzy logic (FL) based angle estimator has been developed with the major objective of having a low sensitivity to feedback signal error and a high robustness. This is achieved with a fuzzy rule based model of the motor which has the ability to be tolerant to input signal noise and error.

A block diagram of the FL based angle estimation algorithm is shown in Fig. 5. The details of the estimation scheme have been previously described ([8], [9]) where actual experimental results were detailed to demonstrate the effectiveness of the method at start-up, low and high speeds, as well as torque and speed transients. In the discussion below the scheme will be briefly outlined, and the robustness of the scheme to noise and error will be detailed.

The estimation algorithm employs a fuzzy logic rule based SR motor model. This rule base is used to provide a value of rotor position from the measured feedback signal inputs. As detailed in [9], the fuzzy rule based model of the SR motor is created with a training scheme that trains the motor model based on measured static and dynamic operation numerical data about the SR motor. After the training, the generated FL rule base defines a nonlinear multi-input single-output function $f$ which maps input values of flux linkage and current to output values of position

$$
f:(\psi, i) \rightarrow \theta
$$




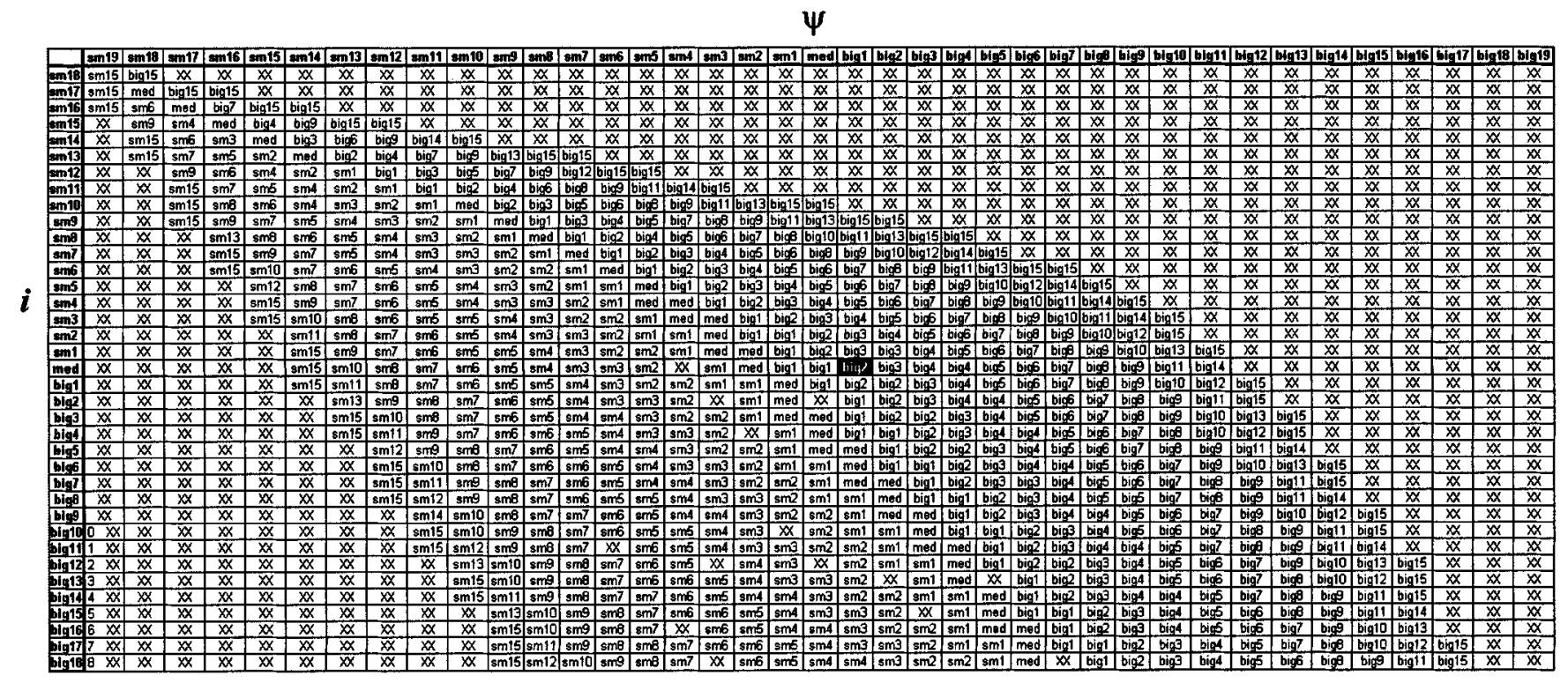

Fig. 6. SR model fuzzy rule base $(\mathrm{sm}=$ small, med $=$ medium, $\mathrm{XX}=$ no rule). HIghlighted cell is the fuzzy set shown in Fig. 7.

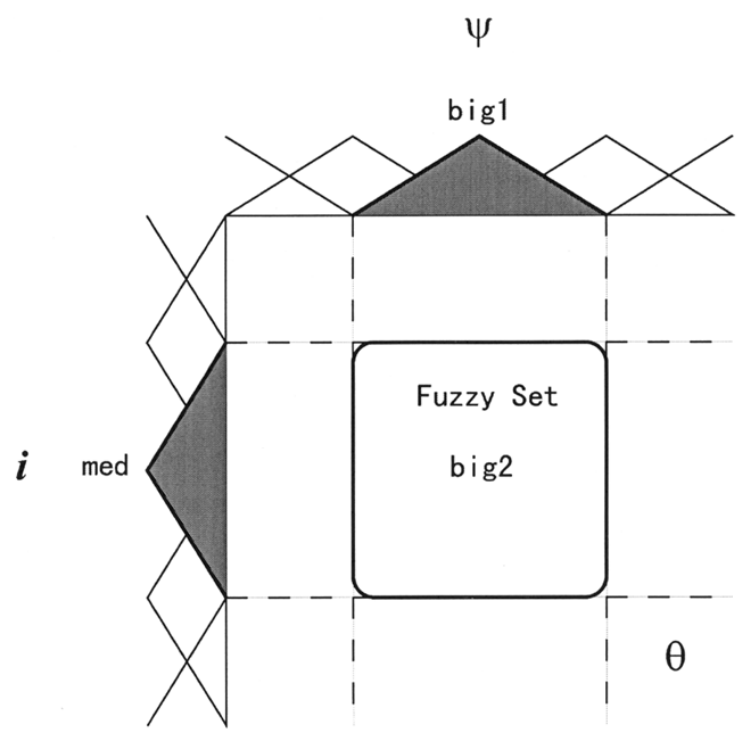

Fig. 7. Expanded subset of SR model fuzzy rule base.

In Fig. 6 results are shown of the generated fuzzy rule base for the motor used in the research, after the rule base was trained with measured data.

The fuzzy rule based model is utilized during the operation of the position estimation scheme to calculate the rotor position from input measurements of current and estimated flux linkage.

First, while the motor is running, the phase currents and voltages in each of the motor phases are measured, and the phase flux linkage is estimated by trapezoidal integration using the motor voltage (2).

After flux linkage estimation, the crisp (or numeric) flux linkage and measured current values of each motor phase are then fuzzified and input into the FL rule based SR motor model. Fuzzy rules in the rule base will be triggered by the crisp inputs, which will then generate a fuzzy set output rotor position. This is then defuzzified to produce a crisp value of estimated rotor position. The hardware and software implementation details of the procedure can be examined in [9].

\section{A. Robustness of Fuzzy Model Based Method}

To examine why there is robustness to noise and error in fuzzy rule based modeling, it should first be noted that each rule of the fuzzy rule base shown in Fig. 6 expresses heuristically the motor characteristics in each region using fuzzy reasoning. Each rule will have the form

$$
R^{(n)}: \text { If } \psi \text { is } A_{\psi}^{(n)} \text { and } i \text { is } A_{i}^{(n)} \text { then } \theta \text { is } B_{\theta}^{(n)}
$$

where

$R^{(n)}$ the $n$th rule;

$A_{\psi}^{(n)}$ antecedent fuzzy set in the flux linkage fuzzy set domain with membership function $\mu_{A_{\psi *}^{(n)}}(\psi)$;

$A_{i}^{(n)}$ antecedent fuzzy set in the current fuzzy set domain with membership function $\mu_{A_{i}^{(n)}}(i)$;

$B_{\theta}^{(n)}$ consequent fuzzy set in the rotor angle fuzzy set domain with membership function $\mu_{B_{\theta}^{(n)}}(\theta)$.

Each of the antecedent and consequent fuzzy sets in the $\psi, i$, and $\theta$ variable domains represent a linguistic variable and are defined over a range of values or a region with membership value ranging from 0 to 1 . In this work the fuzzy sets were defined to be triangular with the maximum value at the center of the fuzzy set's respective region. The two other vertices were chosen to lie at the centers of the two adjacent fuzzy regions, and at these two points the membership values are zero.

Hence for any given rule, both the antecedents and the consequents in the input-output domains will be defined for a given range of values. For example consider a rule from the motor model that was shown in Fig. 6

$$
\text { If } \psi \text { is } b i g 1 \text { and } i \text { is med then } \theta \text { is big2. }
$$

Fig. 7 shows an expanded form of Fig. 6 where this rule is represented. The consequent of the rule is the fuzzy set big2 in 


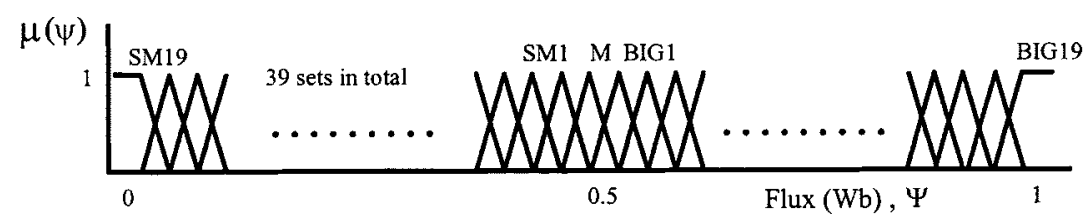

(a)

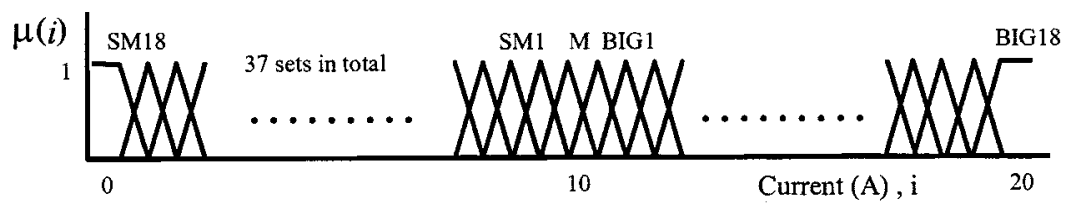

(b)

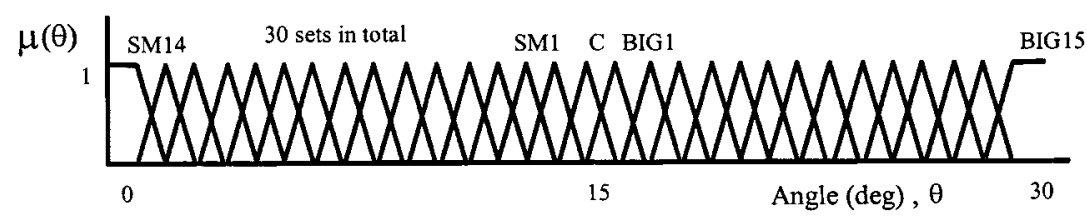

(c)

Fig. 8. Fuzzy variable domain sets: (a) flux linkage, (b) current, and (c) rotor position. Here $\mu(\psi), \mu(i)$, and $\mu(\theta)$ are the membership values of the fuzzy sets in the flux, current, and angle domains, respectively.
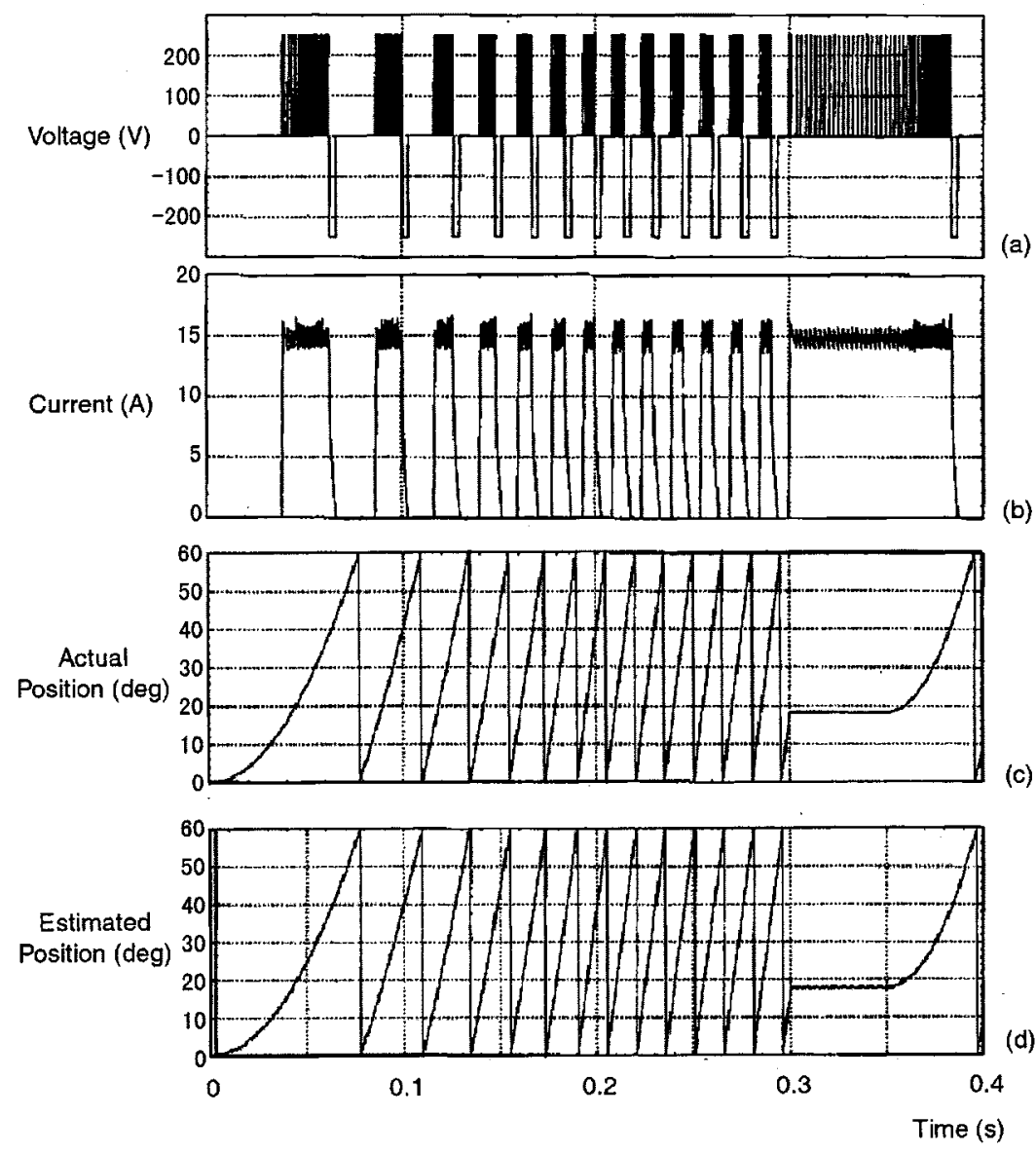

Fig. 9. Waveforms for 0-1000 rpm transient test: (a) phase voltage, (b) phase current, (c) actual position, and (d) estimated position.

the angle domain, and it is triggered by the antecedent fuzzy sets big1 in the flux linkage domain and med (medium) in the current domain.
The width or size of the membership functions of the fuzzy sets can be considered as an allowable level of noise [10], [11]. This means that an input data point with error or noise can still 


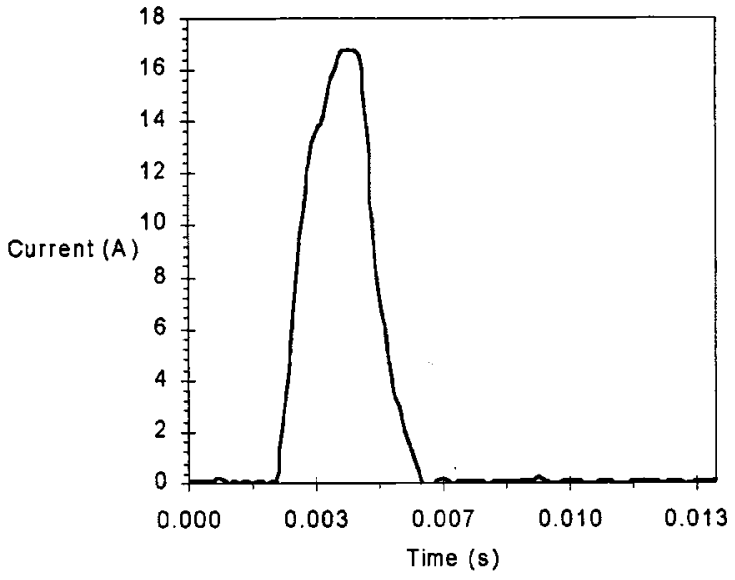

(a)

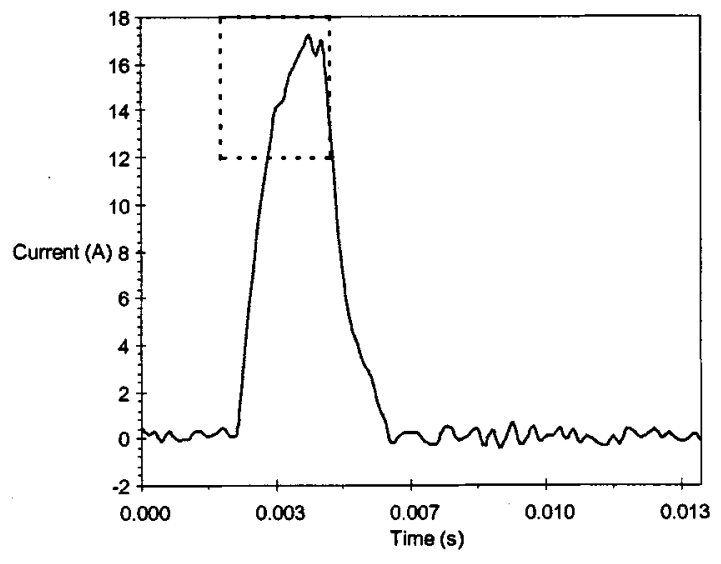

(b)

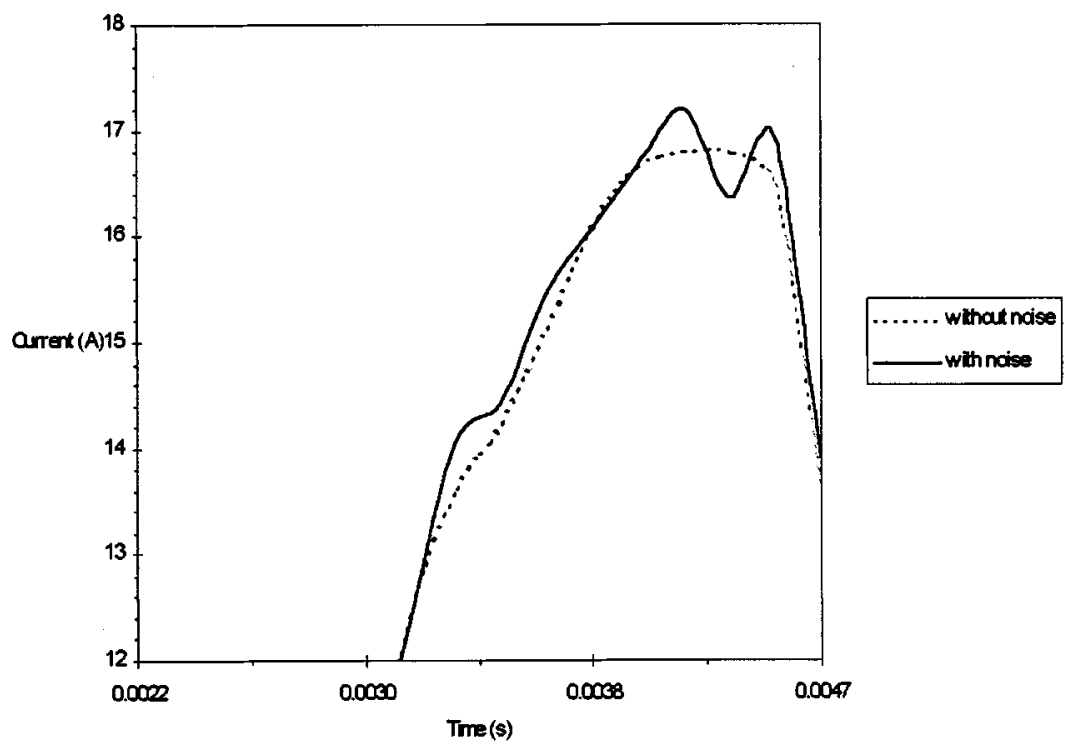

(c)

Fig. 10. SR motor phase current: (a) without noise, (b) with added noise (10\% of maximum), and (c) expanded comparison of current waveforms, with and without noise.

be placed in the same fuzzy set as the same input data with no error or noise. Thus by fuzzification of the input signals, input data which is corrupted by noise can be accepted in the same set as the same data without noise but with a different membership function [12]. The length or range of the fuzzy set membership function will determine the range of values that will be accepted as part of the same set. The range can be defined such that small deviations in the input data do not have a significant effect on the output position estimation.

However, it should also be noted that a disadvantage of using the fuzzy rule based modeling is that an inverse relationship exists between robustness and resolution. As the robustness to input signal noise and error increases due to a widening of the fuzzy sets, the output resolution decreases due to the wider partitioning of the input and output signal domains. Therefore a balance must be found between error robustness and output ac- curacy. In this research work each variable domain of flux, current, and angle was divided up into the fuzzy regions as shown in Fig. 8. These resulted in a good compromise between accuracy and robustness to noise and error.

However, as in all cases where finite width fuzzy sets are used, when there is zero or very small input signal error, the output will normally still contain some error or uncertainty due to the fuzzy set partitioning of the input and output domains. This entails that, strictly speaking, if robustness is not of concern then conventional schemes may result in higher accuracy at zero or no noise levels.

To observe the performance of the scheme with no feedback signal noise (and only a small error due to numerical integration of flux linkage), simulation results are shown in Fig. 9. In the test the average value of error between actual and estimated angle is $0.4^{\circ}$, whereas the nonfuzzy schemes may have little or no error. 


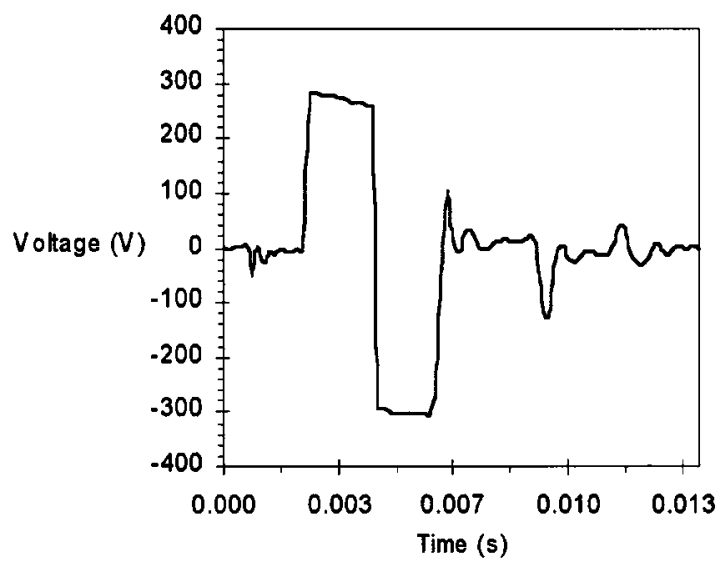

(a)

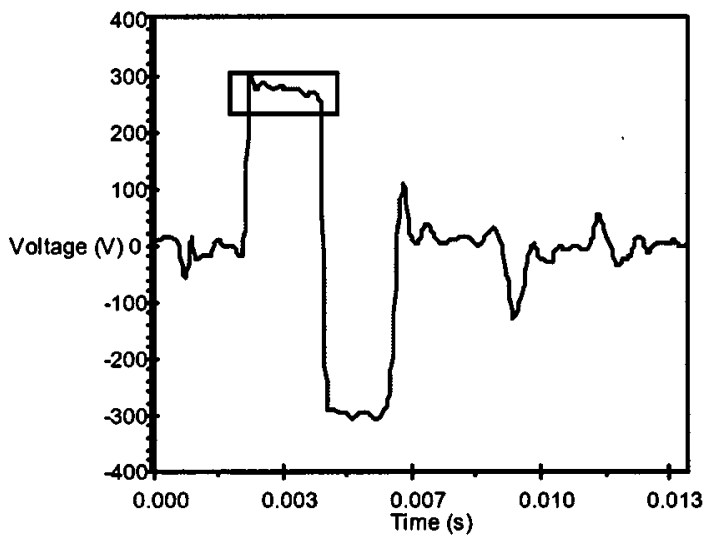

(b)

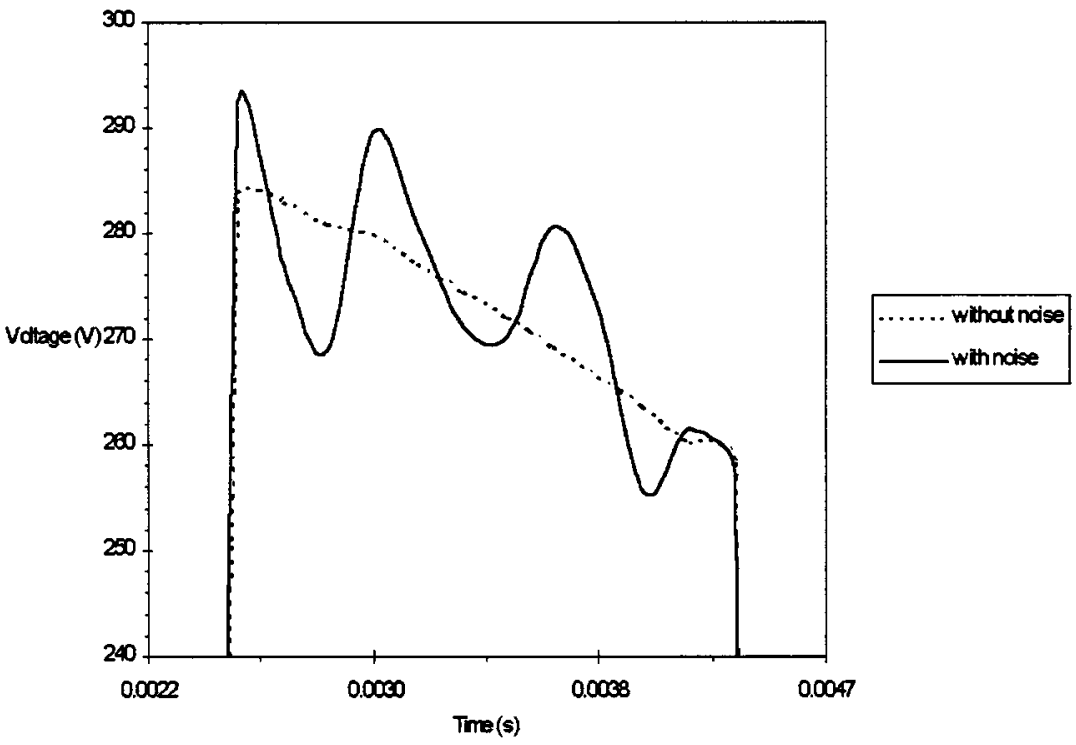

(c)

Fig. 11. SR motor phase voltage: (a) without noise, (b) with added noise (10\% of maximum), and (c) expanded comparison of voltage waveforms, with and without noise.

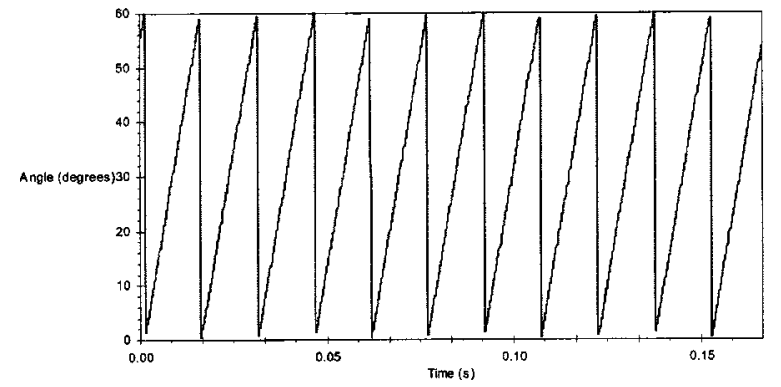

(a)

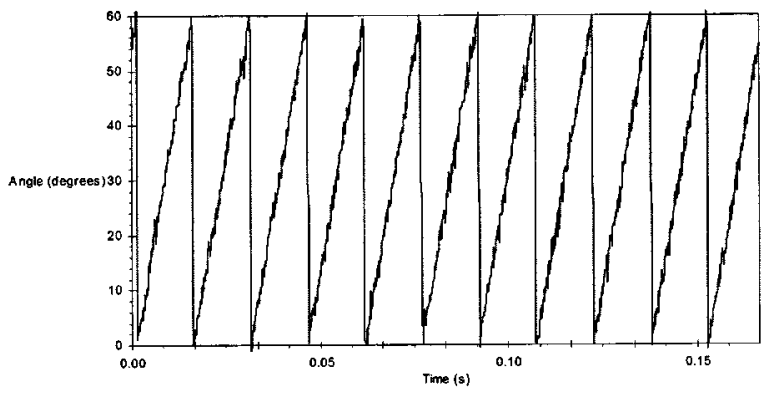

(b)

Fig. 12. Ten percent noise: (a) measured rotor position and (b) estimated rotor position.

This error is mainly due to the above mentioned uncertainty of fuzzy partitioning. To increase the accuracy of the scheme at zero or low feedback error, the number of fuzzy regions may be increased or optimized, at the expense of robustness. The robustness of the scheme will be examined with experimental results in the following section. 


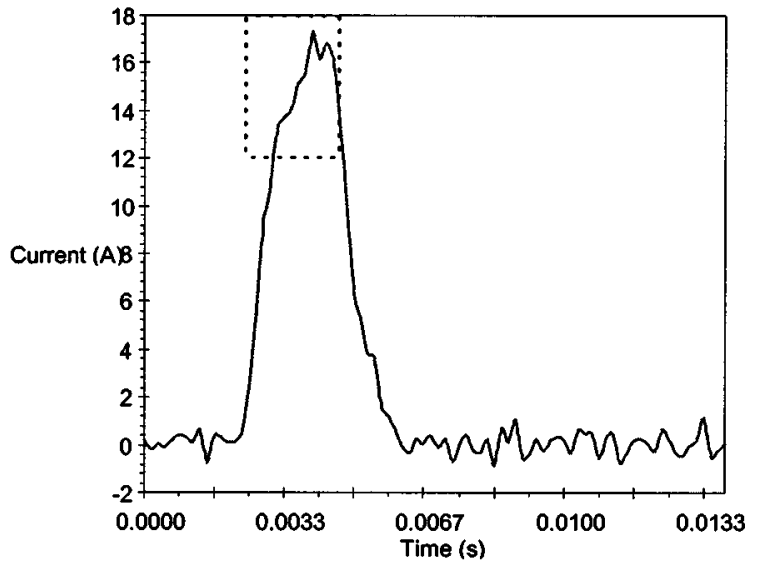

(a)

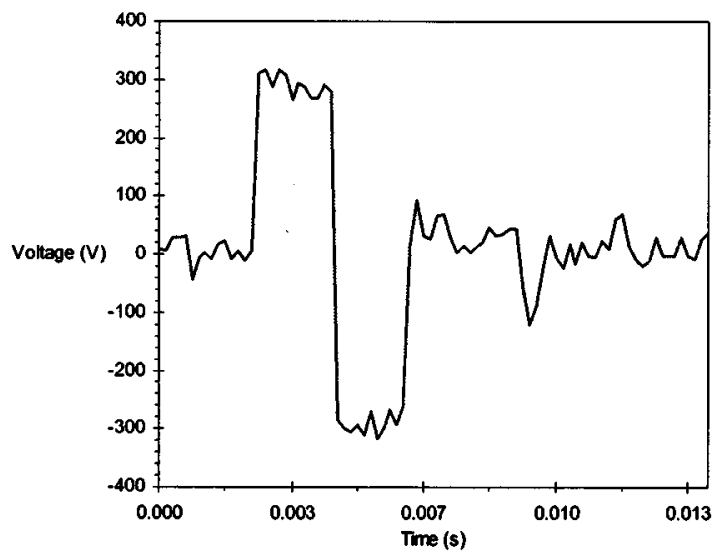

(c)

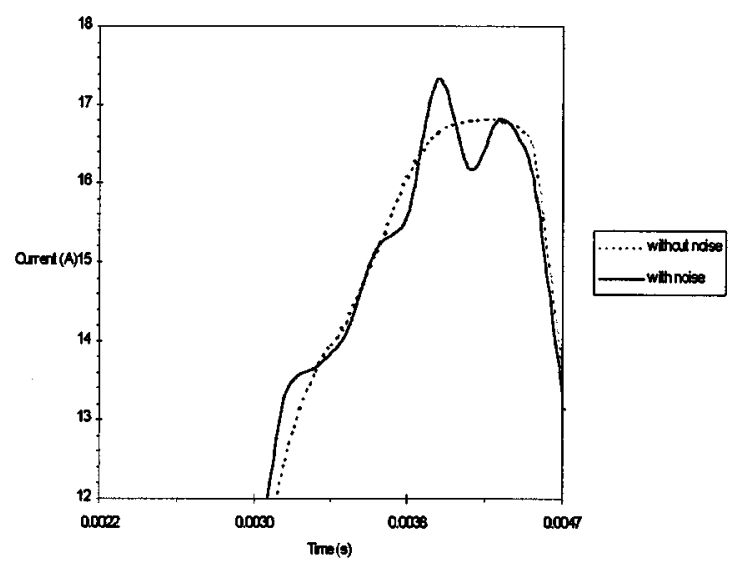

(b)

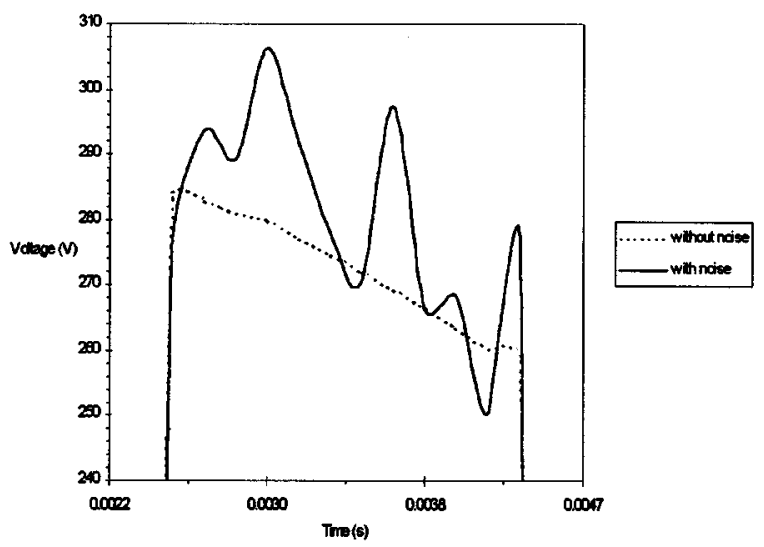

(d)

Fig. 13. Twenty percent of maximum amplitude noise: (a) SR motor phase current, (b) expanded comparison of current waveforms, with and without noise, (c) SR motor phase voltage, and (d) expanded comparison of voltage waveforms, with and without noise.

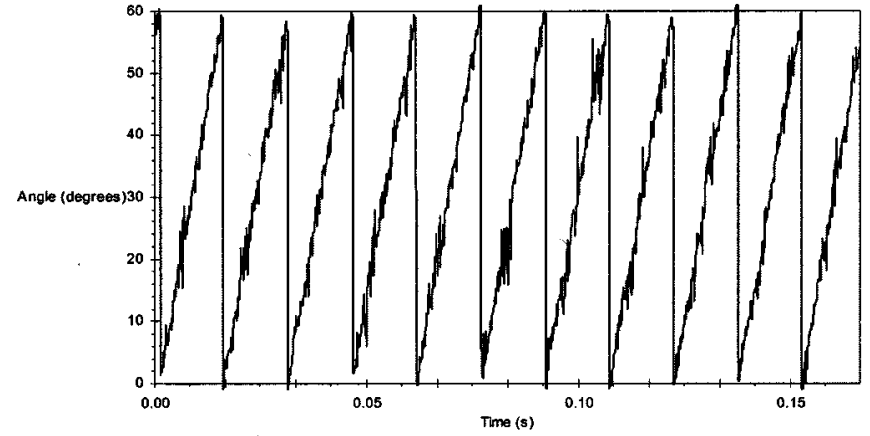

Fig. 14. Twenty percent noise: estimated angle.

\section{EXPERIMENTAL RobUSTNESS TESTS}

To examine the experimental robustness of the angle estimation algorithm, it is firstly instructive to examine the concept of robustness from the field of robust statistics. Huber has defined robust algorithms as having the following features [13].

1) The algorithm should have a reasonably good accuracy in the assumed model of the underlying system.

2) Small deviations of the data should decrease the performance of the algorithm by only a small amount.
3) Large deviations in the data should not cause a catastrophe.

The first of these requirements essentially states that the output of the algorithm should be accurate when the input data is noise free. It was seen in [8], [9], and Fig. 9 that the accuracy of the algorithm in predicting rotor position has a good accuracy for relatively noise free feedback signals of current and voltage. Therefore, the sensorless position detection algorithm has been shown to satisfy the first requirement of a robust algorithm.

To satisfy the second requirement of a robust system entails that small deviations in the inputs of the sensorless algorithm should not have a significant adverse effect on the output rotor position estimate.

Furthermore, to satisfy the third requirement of algorithm robustness, the notion of a breakdown point should be considered [14]. The breakdown point is defined as a point where the algorithm fails by producing an unacceptably high output error due to a large deviation in the input data. A deviation in the input data can be defined as large when it has abnormally high deviation from the correct value, compared to the mean level of input data noise. For example in the SR motor drive there may be high amplitude but short duration periods of electromagnetic noise, due to fast turn off of the current in the inverter power devices. 


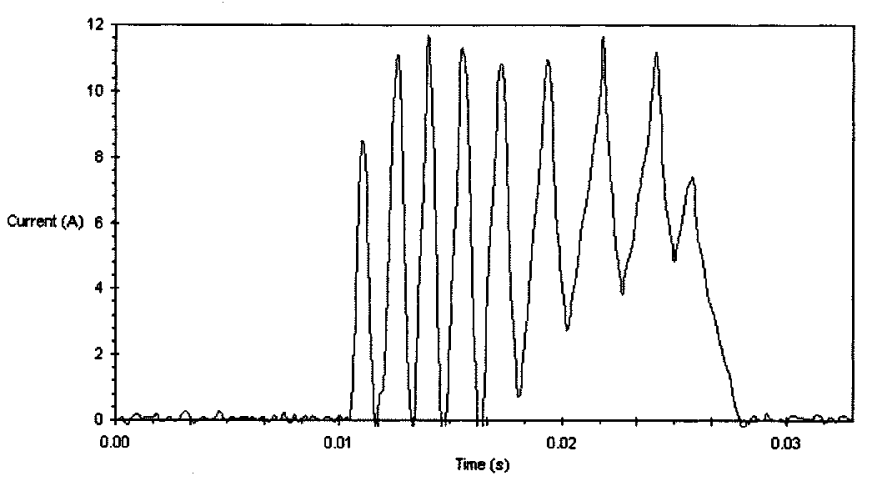

(a)

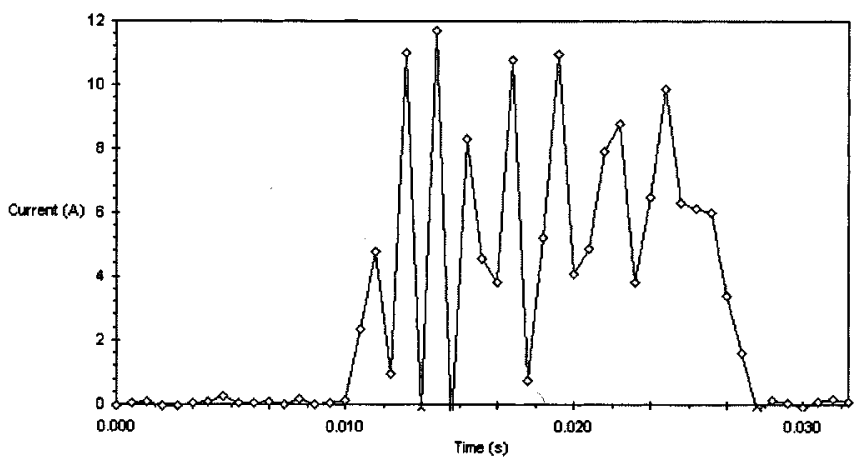

(b)

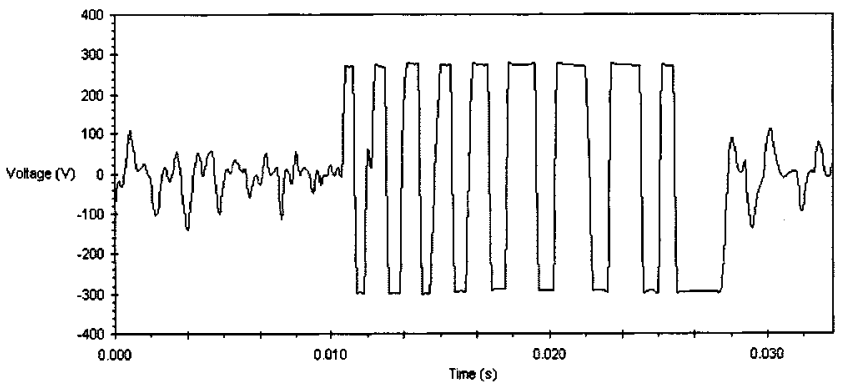

(c)

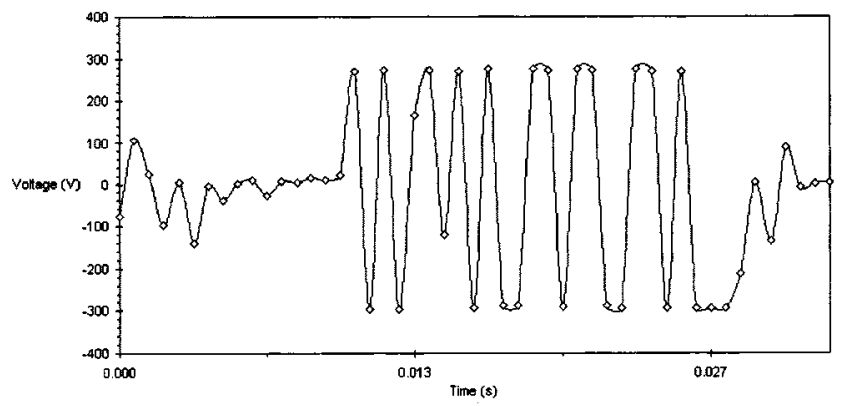

(d)

Fig. 15. (a) Current: $6 \mathrm{kHz}$ sampling and (b) current: $1.5 \mathrm{kHz}$ sampling.

In the field of robust statistics these higher deviations in the data are termed outliers, as opposed to inliers which lie closer to the mean level of noise [15]. Although these robust statistic concepts are directly applicable to engineering tasks such as modeling and pattern recognition, the idea of a breakdown point and outlier and inlier data is applied in the FL based estimation algorithm by testing the algorithm with a very high level of signal noise, as detailed below.

\section{A. Measurement Error Tests}

In Figs. 10 and 11 the waveforms of current and voltage for one phase of the SR motor are shown for the case when the motor is operating in single pulse mode at a steady speed of $660 \mathrm{rpm}$. There is an added random noise error with an amplitude of $10 \%$ of the maximum level of the measured signals. The added noise is Gaussian in nature, and therefore, has a finite variance, (the problem of non-Gaussian noise in this scheme has previously been examined in [7]). The expanded view of the current and voltage in Figs. 10(c) and 11(c) clearly shows that the voltage and current with noise deviates significantly at various points in time from the measured values without added noise.

The measured and indirectly estimated rotor angle for this test is shown in Fig. 12. The average amplitude of the error between the measured and estimated angle in this test is $1.24^{\circ}$, while the maximum amplitude of error is $3.97^{\circ}$. These values represent 2.06 and $6.6 \%$ of one electrical cycle of $60^{\circ}$, respectively. Note that the maximum angle estimation error is less than the $10 \%$ input signal error. Hence, the error in the estimated angle is not high relative to the input error, and operation of the motor is still possible. Therefore the second requirement of a robust algorithm, as described above, has been satisfied in this case.

For comparison, it can be recalled from the results shown in Section II-A for nonfuzzy methods of position estimation, that for a $10 \%$ error in the current feedback input the corresponding minimum position errors ranged from $2.8-15.6 \%$ depending on the flux linkage amplitude. Furthermore a $10 \%$ error in the flux linkage feedback led to minimum position estimation errors ranging from 5.67-63.87\%. Additionally, it was seen that the error increased significantly in other nonoptimum regions in the $(\psi, i, \theta)$ plane. As there are simultaneous errors in both the flux linkage and current feedback in this test, the total error will be a combination of the two errors as expressed in (11).

Therefore, it can be seen that the use of fuzzy sets improves the robustness of position estimation, due to the fact that the $a v$ erage position estimation error using the fuzzy model is of the same order as the minimum error using the nonfuzzy method, and the maximum error of the fuzzy method is significantly lower.

Further experiments were carried out to test the scheme at higher noise levels, and to see the operational limits of the current system. The level of noise imposed on the motor waveforms of current and voltage was increased to $20 \%$ of the input measured signals. It can be seen from Fig. 13 that in this set of results the noise has a significant component, and provides a difficult test for the sensorless algorithm. In Fig. 14 the estimated angle from the angle estimation algorithm is shown for this experiment (the measured angle was shown above in Fig. 12). It can be seen that the general trajectory of the rotor angle has been retained in this test, even for this high level of noise. The average angle estimation error is $2.80^{\circ}(4.67 \%)$, whilst the peak error is $9.92^{\circ}$, or $16.5 \%$ of the electrical angle cycle of $60^{\circ}$. The peak error is lower than the maximum input signal error amplitude. 


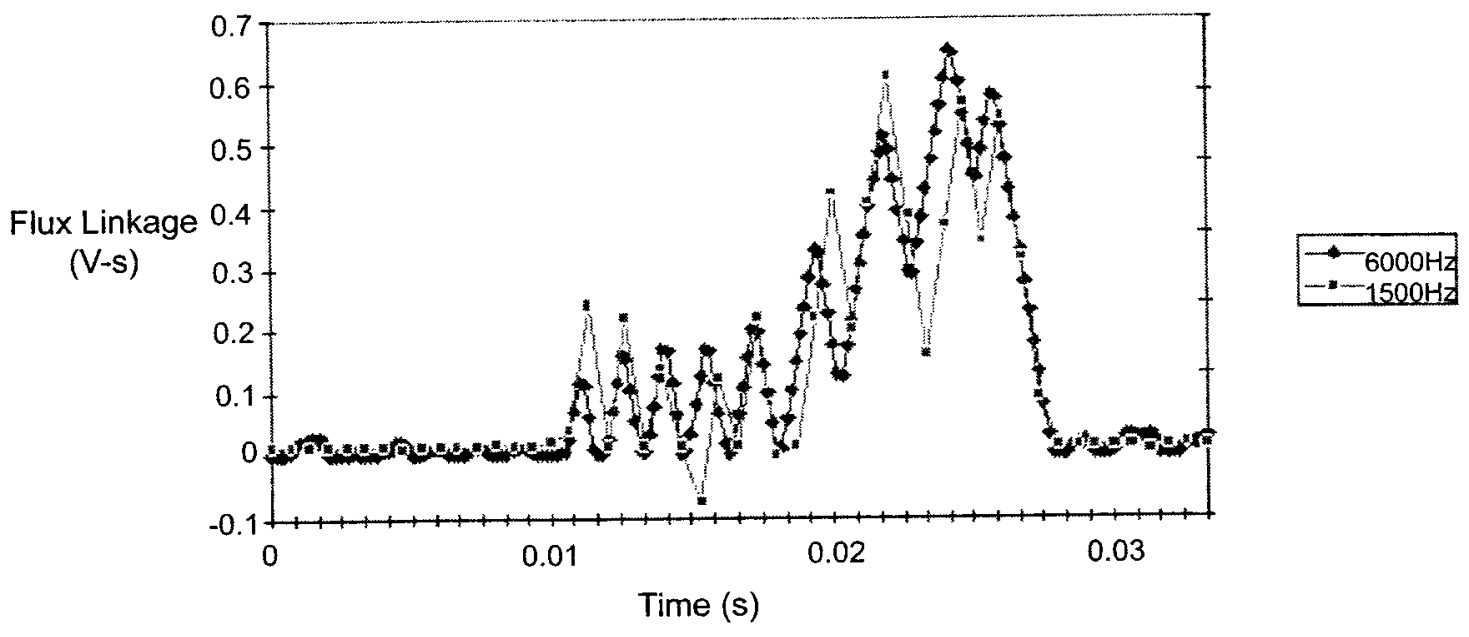

Fig. 16. Comparison of flux linkages derived with 6 and $1.5 \mathrm{kHz}$ sampling frequencies.

Furthermore, at no point in this test has the large deviation of the input current and voltage data caused a catastrophic breakdown of the algorithm, and thus the third requirement of a robust algorithm has also been satisfied.

Hence, the requirements of a robust algorithm have been demonstrated in this test. It has been shown that the large deviations in the input current and voltage do not produce a catastrophic breakdown in the algorithm, and even the peak estimation error has a lower percentage error than the peak input error. In addition, as the average angle estimation error in this high noise test was $2.80^{\circ}$, it entails that the smaller deviations in the noisy inputs produce only small deviations in the output estimated signal.

\section{B. Effect of Low Sampling Rate}

In this section, another important practical effect in the operation of the angle estimation scheme in real SR motor drives is considered. During operation with a practical SR motor drive, the sampling rate of the analog to digital converters, which convert the measured waveforms into digital data, will have some effect on the accuracy of the position estimation due to amplitude and time quantization. A system that is only accurate with high sampling rates may not be useful in a practical application. This is because expensive high sampling rate A/D converters may not suit a particular application in which the total system cost must be kept low, or may not be compatible with lower speed processors (e.g., microcontroller based drives).

Therefore, various tests were performed using experimental data, to determine the ability of the sensorless scheme to operate at low sample rates. In these tests, the sampling frequency was deliberately reduced. This entails that the theoretical required processing speed of the algorithm also becomes lower, because the rotor angle is estimated only once per sampling period (when new information about the voltage and current magnitudes is entered into the algorithm). Hence, the results in this section will demonstrate that the scheme is viable also in systems with a low A/D converter sampling speed and a low speed microprocessor or microcontroller.
Flux linkage is one of the inputs to the fuzzy model in the sensorless angle estimation algorithm. It can be recalled from Table II that the numerical flux linkage integration becomes less accurate when the sampling rate is reduced due to the time quantization of the $\mathrm{A} / \mathrm{D}$ converter error. The accuracy in the flux linkage integration is particularly affected at low sampling frequencies. Hence, the major effect of low sampling rates on the angle estimation algorithm, is the decrease in accuracy of the numerical flux linkage integration which is used as an input to the position estimator.

In Fig. 15, the experimental results of current and voltage are shown for a sampling frequency of $1.5 \mathrm{kHz}$, which represents a sampling period of $667 \mu \mathrm{s}$, or 28 samples per current pulse. It can be seen from the comparison of the current waveform sampled at $1.5 \mathrm{kHz}$, and that sampled at $6 \mathrm{kHz}$, that a significant amount of the dynamic characteristic of the waveform are lost due to the low sampling rate.

A sampling frequency of at least $5-10 \mathrm{kHz}$ is generally required for digital control of electric machines. Therefore, a sampling frequency of $1.5 \mathrm{kHz}$ would not normally be feasible in a practical digital motor control system, and thus this test would be at the lower limit of the possible sampling frequencies.

Due to the reduction in sampling frequency, the flux estimation has a higher error component. One can see this by comparing the estimated flux at $1.5 \mathrm{kHz}$ in Fig. 16 with the flux estimate calculated at $6 \mathrm{kHz}$ sampling frequency. In this test, the peak error between the flux estimated at $1.5 \mathrm{kHz}$ sampling frequency is $29.8 \%$ of the peak flux amplitude measured at $6 \mathrm{kHz}$ (which in itself has some integration error).

In Fig. 17 the result of the angle estimation for this low frequency test is shown, where one can see that, although there is error in the angle estimate, the general path of the rotor angle is followed throughout the test. The average error in the results was $3.82^{\circ}(6.37 \%)$, whilst the maximum error was $7.79^{\circ}$, or $13 \%$ of one electrical cycle. Thus even with very low sampling frequency and flux estimation error, there is no breakdown of the algorithm, and hence the robustness of the algorithm to low sampling frequencies is demonstrated. 


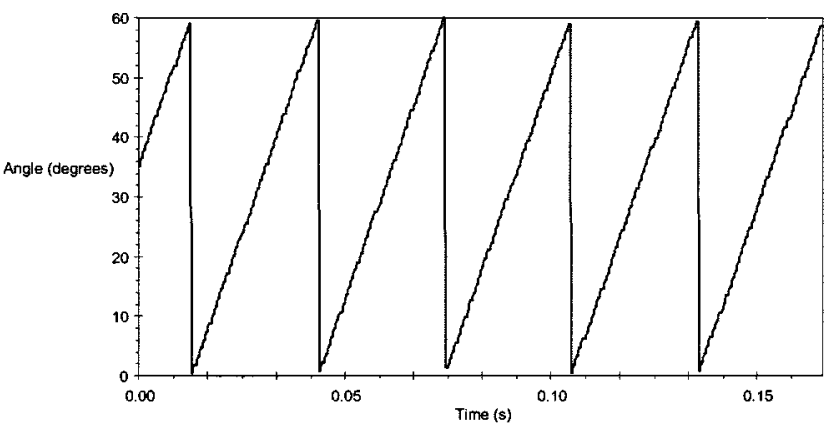

(a)

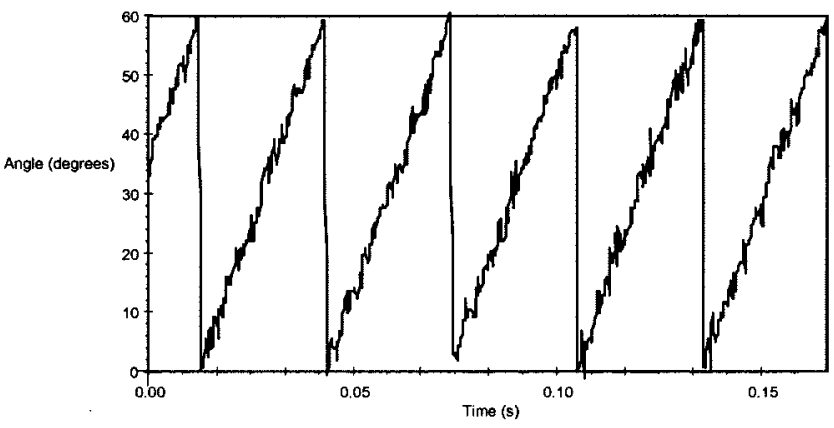

(b)

Fig. 17. Sampling frequency at $1.5 \mathrm{kHz}$ : (a) measured angle and (b) estimated angle.

\section{Flux Linkage Offset Error}

As detailed above, the flux linkage of each motor phase is estimated by integration using the measured phase voltages and phase currents. In the SR motor, the flux always returns to zero at the end of each current conduction period, which allows the integrator to be reset in every electrical cycle. This prevents any large accumulation of errors due to the effects of current and voltage dc offset, measurement errors, and resistance variation. For this reason, the flux estimation technique is very effective in the SR motor.

Although the flux linkage integration is reset in each electrical cycle, to test the robustness of the angle estimation algorithm, results are shown below for operation with flux linkage waveforms which have a constant offset error.

Fig. 18(a) shows the flux linkage for the case when the motor is operating in the chopping mode with a speed of $162 \mathrm{rpm}$. In this test a flux linkage error is added such that the flux linkage has a constant positive offset of $0.025 \mathrm{Vs}$, or $4 \%$ of the peak flux linkage value in this test. Although this is not an overly large error value, due to the fact that is has a constant value, it means that the relative error effect at the lower flux values is higher than for the peak flux values.

The estimated rotor position for this test is shown together with the actual angle in Fig. 18(b) and (c). The average angle estimation error in this test is $1.47^{\circ}(2.45 \%)$, whilst the peak error is $6.0^{\circ}$, or $10 \%$ of one electrical cycle. Even though there is a constant flux linkage error, the angle estimation error is not exceedingly large and this highlights the ability of the scheme to operate with corrupted flux linkage signals.

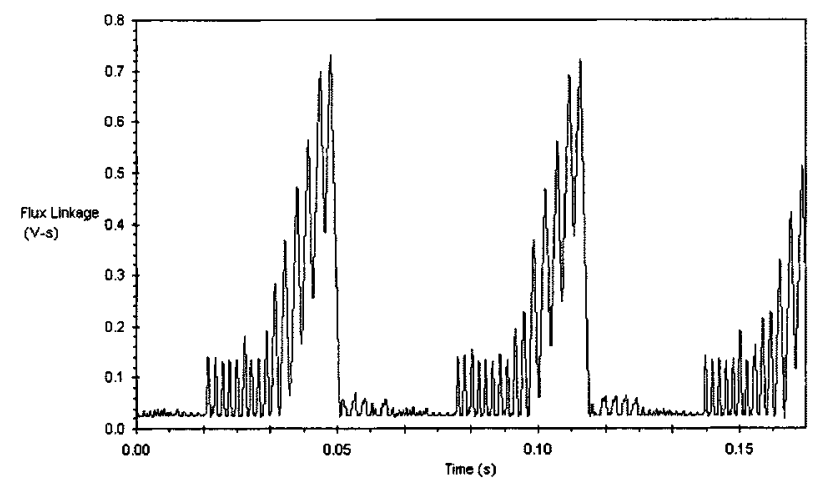

(a)

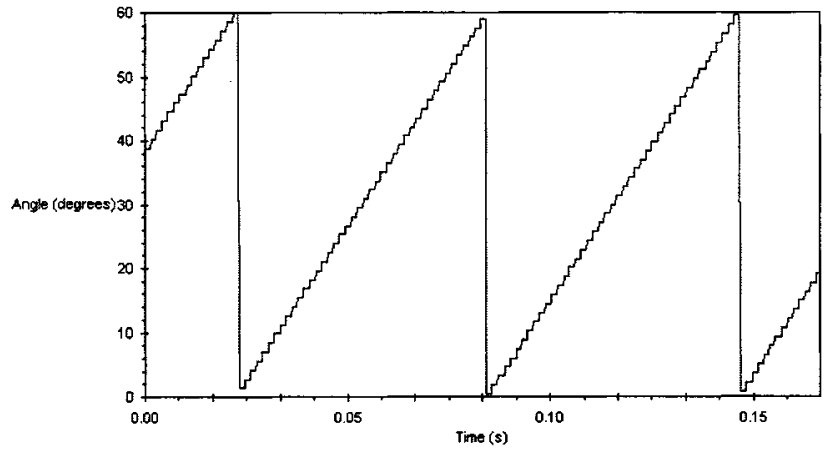

(b)

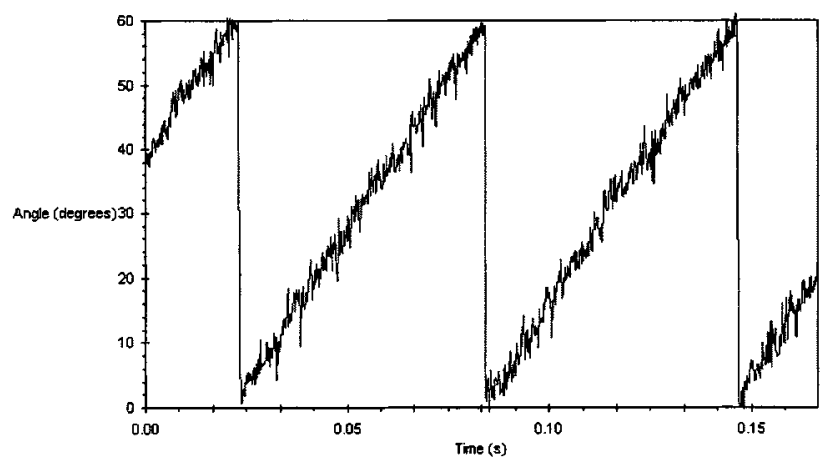

(c)

Fig. 18. (a) Flux linkage with offset of $25 \mathrm{mWb}$, (b) measured angle, and (c) estimated angle.

\section{CONCLUSION}

In most applications where motor drives are used, the reliability of the drive is of utmost concern. This is particularly the case for some applications of the SR motor drive, such as in aerospace type applications [16], where the reliability and robustness (such as the ability to operate when one or more phases fail) are the main reasons for the choice of the motor drive. Although it is commonly stated that position sensors may have the effect of reducing the reliability of the motor, position sensorless control methods may also reduce the system reliability unless their robustness in practical motor drive operation is proven.

In this paper, it was shown using theoretical explanations and experimentally based results, that the fuzzy logic based angle 
estimation scheme has a high level of robustness and reliability, and is thus well suited for a wide range of practical systems. Results were detailed which showed that the scheme could successfully and reliably operate under erroneous operating conditions that are commonly found in motor drive environments as follows.

1) Measurement errors due to noise.

2) Low bandwidth motor waveform measurements (e.g., due to slower analog to digital converters).

3) Flux linkage estimation errors, which may be caused by:

a) Offset errors.

b) Measurement errors.

c) Noise.

In order to compare the results of the fuzzy logic based method with nonfuzzy angle estimation, analytical expressions were given which described the effect of current and flux linkage input errors on the angle estimation accuracy of nonfuzzy schemes. Then the position estimation errors were calculated for nonfuzzy estimation. Hence, it was confirmed that the average and maximum position estimation errors of the fuzzy logic based estimation scheme due to feedback signal error, were low compared to the nonfuzzy estimation methods.

The ability of the FL based method to cope with high levels of noise derives from the system's ability of fuzzifying the input signals and then processing with fuzzy linguistic rules. With this system, an input with noise will normally be able to be partially a member of the same membership function that the error free signal would be. This is in fact a unique ability of fuzzy logic, as opposed to crisp logic. Therefore, the same rules can be triggered by noisy and noise free signals, depending on the level of the noise. Hence, using the benefits of fuzzy reasoning rotor position can be successfully and reliably estimated under high error and noise conditions in practical SR drives.

\section{APPENDIX A \\ EXPERIMENTAL MOTOR DATA}

Number of phases 4

Number of rotor poles

6

Number of stator poles

Rated power

$4 \mathrm{~kW}$

Rated speed

$1500 \mathrm{rpm}$

\section{REFERENCES}

[1] A. D. Cheok and N. Ertugrul, "Sensorless rotor position detection techniques in switched reluctance motor drives," in Proc. Australasian Univ. Power Eng. Conf., 1995, pp. 84-89.

[2] W. F. Ray and I. H. Al-bahadly, "Sensorless methods for determining the rotor position of switched reluctance motors," in Proc. 5th Eur. Conf. Power Electron. Appl., 1993, pp. 7-13

[3] P. Acarnley, E. D. French, and I. H. Al-bahadly, "Position estimation in switched reluctance drives," in Proc. 7th Eur. Conf. Power Electron. Applicat., vol. 3, 1995, pp. 765-770.

[4] I. Husain and M. Ehsani, "Error analysis in indirect rotor position sensing of switched reluctance motors," IEEE Trans. Ind. Electron., vol. 41, pp. 301-307, June 1994.
[5] S. Rehman and D. G. Taylor, "Issues in position estimation of sr motors," in IEEE Power Electron. Spec. Conf. Rec., 1996, pp. 337-343.

[6] T. Williams and R. Carter, "Measurement of machine inductances using an operational amplifier integrator," Int. J. Elect. Eng. Educ., vol. 10, pp. $177-181,1972 / 73$

[7] A. D. Cheok and N. Ertugrul, "High robustness and reliability of a fuzzy logic based angle estimation algorithm for practical switched reluctance motor drives," in Proc. IEEE Power Electron. Specialists Conf. Rec., 1998, pp. 1302-1308.

[8] _ - "A model free fuzzy logic based rotor position sensorless switched reluctance motor drive," in Proc. IEEE Ind. Appl. Soc. Annu. Meeting, 1996, pp. 76-83.

[9] N. Ertugrul and A. D. Cheok, "Indirect angle estimation in switched reluctance motor drives using fuzzy logic based predictor/corrector," in Proc. IEEE Power Electron. Spec. Conf. Rec., 1998, pp. 845-851.

[10] P. J. Costa Branco and J. A. Dente, "An experiment in automatic modeling an electrical drive system using fuzzy logic," IEEE Trans. Syst., Man, Cybern. C, vol. 28, pp. 254-262, May 1998.

[11] G. C. Mouzouris and J. M. Mendel, "Dynamic non-singleton fuzzy logic systems for nonlinear modeling," IEEE Trans. Fuzzy Syst., vol. 5, pp. 199-208, May 1997.

[12] J. van den Berg and D. Ettes, "Representation and learning capabilities of additive fuzzy systems," in Proc. 1998 IEEE Int. Conf. Intell. Eng. Syst., 1998, pp. 121-126.

[13] P. J. Huber, Robust Statistics. New York: Wiley, 1981.

[14] R. N. Dave and R. Krishnapuram, "Robust clustering methods: A unified view," IEEE Trans. Fuzzy Syst., vol. 5, no. 2, pp. 270-293, 1997.

[15] F. R. Hampel, E. M. Ponchotti, P. J. Rowsseuw, and W. A. Stahel, Robust Statistics: The Approach Based on Influence Functions. New York: Wiley, 1986.

[16] A. V. Radun, C. A. Ferreira, and E. Richter, "Two-channel switched reluctance starter/generator results," IEEE Trans. Ind. Applicat., vol. 34, no. 5 , pp. 1026-1034, Sept./Oct. 1998.

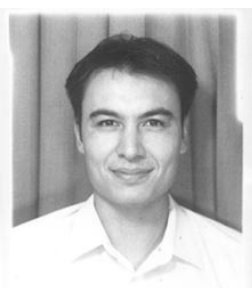

Adrian David Cheok (M'92) received the B.Eng. (first class honors) and Ph.D. degrees from the University of Adelaide, Australia, in 1993 and 1998, respectively.

From 1996 to 1998, he was with the Transmission and Distribution Department, Transportation Systems Center,Mitsubishi Electric Corporation, Amagasaki, Japan. Since 1998, he has been an Assistant Professor in the Department of Electrical Engineering, National University of Singapore. His research interests include power electronics and motor drives, fuzzy logic and soft computing, nonlinear modeling and control, noise and EMI, and digital signal processing.

Dr. Cheok is a Member of the IEEE Industrial Electronics Society and the IEEE Systems, Man, and Cybernetics Society.

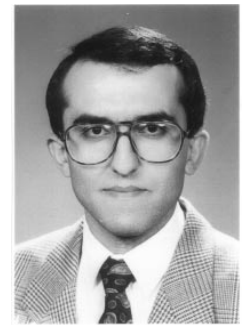

Nesimi Ertugrul (M'95) received the B.Sc. degree in electrical engineering and the M.Sc. degree in electronic and communication engineering, both from the Istanbul Technical University, Istanbul, Turkey, in 1985 and 1989, respectively, and the Ph.D. degree from the University of Newcastle upon Tyne, U.K., in 1993.

He joined the University of Adelaide in1994. His research interests include rotor position sensorless operation of brushless permanent magnet and switched reluctance motors, real-time control of electrical machine drives, electric vehicles, and power electronics utility systems. He is currently engaging in research in the field of interactive computer-based teaching and learning systems involving object-oriented programming and data acquisition.

Dr. Ertugrul is a Member of the IEEE Power Electronics Society and the IEEE Industry Applications Society. 